\title{
DNA methylation is involved in the pathogenesis of osteoarthritis by regulating CtBP expression and CtBP-mediated signaling
}

\author{
Xiangxiang Sun, Lin Xiao, Juan Chen, Xun Chen, Xinlin Chen, Shuxin Yao, Hui Li, Guanghui Zhao, and \\ Jianbing Ma $\bowtie$ \\ Department of Orthopaedics, Honghui Hospital, Xi'an Jiaotong University, Xi'an 710054, Shaanxi, China. \\ $\bowtie$ Corresponding author: Jianbing Ma. E-mail: zeltasun@163.com. \\ (c) The author(s). This is an open access article distributed under the terms of the Creative Commons Attribution License (https://creativecommons.org/licenses/by/4.0/). \\ See http:/ /ivyspring.com/terms for full terms and conditions.
}

Received: 2019.09.03; Accepted: 2019.12.26; Published: 2020.01.30

\begin{abstract}
Osteoarthritis (OA) is a common type of arthritis. Chronic inflammation is an important contributor to the pathogenesis of $O A$. The maturation and secretion of proinflammatory cytokines are controlled by inflammasomes, especially NLRP1 (NLR Family Pyrin Domain Containing 1) and NLRP3. In this study, we identified a transactivation mechanism of NLRP3 mediated by CtBPs (C-terminal-binding proteins). We found that both the mRNA and protein levels of CtBPs were significantly increased in OA biopsies. Analyzing the profiles of differentially expressed genes in CtBP-knockdown and overexpression cells, we found that the expression of NLRP3 was dependent on $C t B P$ levels. By the knockdown or overexpression of transcription factors that potentially bind to the promoter of NLRP3, we found that only API could specifically regulate the expression of NLRP3. Using immunoprecipitation (IP) and Co-IP assays, we found that API formed a transcriptional complex with a histone acetyltransferase $\mathrm{p} 300$ and CtBPs. The knockdown of any member of this transcriptional complex resulted in a decrease in the expression of NLRP3. To explore the underlying mechanism of $C t B P$ overexpression, we analyzed their promoters and found that they were abundant in $C_{p G}$ islands. Treatment with the DNA methylation inhibitor 5-aza-2'deoxycytidine (AZA) or knockdown of DNMTs (DNA methyltransferases) resulted in the overexpression of CtBPs, while overexpression of DNMTs caused the reverse effects on CtBP expression. Collectively, our results suggest that the decreased DNA methylation levels in the promoters of $C t B P s$ upregulate their expression. Increased CtBPs associated with $\mathrm{p} 300$ and API to form a transcriptional complex and activate the expression of NLRP3 and its downstream signaling, eventually aggravating the inflammatory response and leading to the pathogenesis of $O A$.
\end{abstract}

Key words: DNA methylation, CtBP, p300, AP1, NLRP3, IL-1B.

\section{Introduction}

Osteoarthritis (OA) is a chronic disorder caused by damage or degeneration of cartilage in joints $[1,2]$. It is estimated that nearly $10 \%-15 \%$ of the population over 60 years old have different degrees of OA, which often leads to stiffness, pain, impaired movement and even severe disability [3]. OA was originally considered as a noninflammatory arthritis because early findings observed fewer leukocytes in OA synovial fluid in comparison to those in other arthritis, such as rheumatoid arthritis (RA), reactive arthritis and septic arthritis [4]. However, recent investigations have revealed that inflammation is strongly implicated in the pathogenesis of OA [4]. Proinflammatory cytokines such as IL-1 $\beta$ (Interleukin 1 beta) [5], IL-6 [6], and TNF- $\alpha$ (tumor necrosis factoralpha) [7], chemokines such as CCL2 (chemokine ligand 2) and CCL20 [8,9], and other inflammatory mediators such as inflammasome members NLRP1 
(NLR Family Pyrin Domain Containing) and NLRP3 are elevated or activated in OA patients [10]. Of these inflammatory cytokines, IL-1 $\beta$ has been well investigated, and it can induce the expression of multiple genes, such as MMP-1 (Matrix Metallopeptidase 1), MMP-3, MMP-8, MMP-13, and COL1A2 (Type II collagen), in OA cartilages [11]. Inflammasome NLRP3 can activate Caspase-1, which further cleaves pro-IL-1 $\beta$ to promote its maturation and secretion [12]. In addition, multiple signaling pathways are activated in the pathogenesis of OA. For instance, the activation of the TLR4 (Toll-like receptor 4)/NF-кB (Nuclear Factor Kappa B) signaling pathway can regulate the expression of numerous cytokines, including IL-6, IL-8, IL-9 and IL-15 [13]. The activation of ROS (reactive oxygen species)-mediated signaling in cartilage can lead to chondrocyte damage and cell death [14]. Wnt/ $\beta$-catenin signaling is also activated and associated with OA progression and severity [15]. Inhibition of Wnt/ $\beta$-catenin signaling can decrease the progression of OA and improve its severity [15]. A variety of these activated signaling pathways eventually affect gene expression in the nucleus by mediating transcription factors $[16,17]$.

Gene transcription is precisely controlled by transcriptional complexes, which commonly include transcription factors [e.g., NF-кB, AP-1 (Activator protein 1), CREB (cyclic AMP response elementbinding protein) and STATs (signal transducers and activators of transcription)], coactivators [e.g., histone acetyltransferase p300 and CBP (CREB binding protein)] and corepressors [e.g., NcoR1 (Nuclear receptor corepressor 1$)$ and CtBPs (C-terminal-binding proteins)] [18-21]. The transcriptional complexes then promote or block the recruitment of RNA polymerase II to the promoters of specific genes [22]. A number of

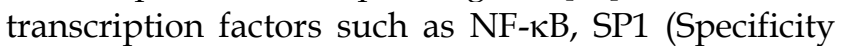
protein 1), KLF4 (Kruppel like factor 4), JDP2 (Jun dimerization protein 2), and c-MYC have been found to participate in the regulation of genes involved in the pathogenesis of OA [23-26]. Histone acetyltransferases (HATs) function to acetylate histone proteins, which impairs DNA-histone associations to make DNA more accessible by transcription factors, thereby inducing gene expression [27-29]. Transcriptional corepressors like CtBPs mainly function to repress gene expression by directly interacting with HATs or HDACs (histone deacetylases) [30, 31]. The human genome contains two CtBP members, CtBP1 and CtBP2, which share nearly $80 \%$ protein sequence identity [32]. The current understanding is that CtBPs have unique roles in different biological processes. CtBP1 functions as an oncogene and is overexpressed in a variety of cancers
[32]. Overexpression of CtBP1 can downregulate multiple tumor suppressors, such as BRCA1 (Breast cancer susceptibility gene 1), CDH1 (Cadherin 1), Bax (BCL2-associated X), Bim (Bcl-2 interacting mediator of cell death), and PTEN (phosphatase and tensin homolog) [32]. CtBP2 is also overexpressed in some cancer types, such as gastric cancer and prostate cancer [33, 34]. Our recent findings revealed that CtBP2 could form a transcriptional complex with p300 and the transcription factor Runx2 (Runt-related transcription factor 2), thereby regulating the expression of many genes involved in bone development and differentiation [35]. Although CtBPs can interact with multiple partners, they have a conserved mechanism in which CtBPs bind other proteins through a short motif known as the PXDLS (Proline-X-Aspartate-Leucine-Serine, where $X$ represents any amino acid) motif [32]. Several studies have reported that in addition to their inhibitory roles, CtBPs have transactivation abilities to regulate gene expression [36-38]. For example, CtBP2 associates with KLF8 (Kruppel-like factor 8) to activate the expression of Tiam1 (T-cell lymphoma invasion and metastasis 1 ), thereby promoting cancer cell migration [36]. CtBP1 is involved in the transactivation of MDR1 (Multidrug Resistance 1) in human multidrugresistant cancer cells [37]. CtBP1 associates with LSD1 (Lysine Demethylase 1), RREB1 (RAS-responsive element-binding protein 1), PCAF (P300/CBPassociated factor) and CoREST (REST Corepressor 1) to form a complex, which can activate the expression of NeuroD1 in gastrointestinal endocrine cells [38]. Thus, CtBPs have both transrepression and transactivation roles in the regulation of gene expression, which is indicative of their complicated roles. Although CtBPs are differentially expressed in many diseases, the molecular mechanisms of their overexpression are still unclear. Some microRNAs (miRNAs) are involved in the regulation of CtBPs and their downstream events. For example, miR-137 functions as a tumor suppressor and it can target $C t B P 1$ directly to inhibit EMT (epithelialmesenchymal transition) and induce apoptosis in melanoma cells [39]. Moreover, miR-212 targets CtBP1 in human endometrial epithelial cells to enhance spheroid attachment in vitro [40].

Recently, we found that $C t B P 2$ but not $C t B P 1$ was overexpressed in atrophic nonunion tissues [35]. The amplified CtBP2 assembled a transcriptional complex with p300, and Runx2 [35]. This complex could medicate the expression of multiple genes, such as COL1A1 (Collagen 1a1), OSC (Osteocalcin), IBSP (Integrin binding sialoprotein), ALPL (Alkaline phosphatase), MMP9 (Matrix metallopeptidase 9), MMP13, and SPP1 (Osteopontin) [35]. To explore if 
CtBPs function in the pathogenesis of OA, we examined the expression levels of $C t B P s$ in $48 \mathrm{OA}$ specimens. Our results showed that both $C t B P 1$ and $C t B P 2$ were significantly upregulated. By knocking down and overexpressing CtBPs in vitro and then analyzing differentially expressed genes that were dependent on CtBPs using a microarray analysis, we found that the expression of NLRP3 was changed with $C t B P$ overexpression or downregulation. Therefore, we will investigate how CtBPs activate NLRP3 and explore the molecular mechanism of $C t B P$ overexpression in this study.

\section{Materials and Methods}

\section{Cell lines and cell culture}

Human osteoarthritic chondrocyte (HC-OA, \#402OA-05A) and human osteoarthritic osteoblast (HOB-OA, \#406OA-05A) cell lines were obtained from Sigma-Aldrich (St. Louis, MO, USA). HC-OA cells are derived from the human articular cartilage of a donor with OA. HOB-OA cells are isolated from the bone of an OA patient. HC-OA cells were cultured in chondrocyte growth medium (PromoCell, Heidelberg, Germany, \#C-27101) supplemented with $10 \%$ fetal bovine serum (FBS) (Sigma-Aldrich, \#F9423), 1\% penicillin-streptomycin (PS) (ThermoFisher Scientific, Waltham, MA, \#10378016). HOB-OA cells were grown in HOB Basal Medium (Cell Application, Inc., San Diego, CA, USA, \#416-500) supplemented with 10\% FBS and 1\% PS. Cells were placed in a $37^{\circ} \mathrm{C}$ humidified incubator containing $5 \%$ $\mathrm{CO}_{2}$.

\section{Biopsy sample collection}

Cartilage tissues were obtained from 48 OA patients who had severe symptoms and needed surgical treatments to replace cartilage by an artificial endoprosthesis. At the same time, biopsies were also collected from another group of patients $(n=48)$ who had no OA symptoms but damaged their joints in accidents. All of these patients were treated at the Department of Orthopedics of Honghui Hospital during the period 2012-2016. The biopsy samples were acquired with written informed consent from patients following a protocol reviewed and approved by the ethical board of Xi'an Jiaotong University. The basic information of these patients is included in Supplementary Table-1.

\section{Total RNA isolation and quantitative real-time PCR (qRT-PCR) analysis}

Total RNA was isolated from tissues and cultured cells using TRIzol (Sigma-Aldrich, \#T9424) following a protocol provided by the manufacturer. The purified RNA $(1.0 \mu \mathrm{g})$ was used to synthesize
cDNA using a PrimeScript ${ }^{\mathrm{TM}}$ 1st strand cDNA Synthesis Kit (TaKaRa, Dalian, China, \#6110B). The resulting cDNA was diluted 10 -fold and then used as a template to detect individual gene expression by qRT-PCR with a TB Green ${ }^{\circledR}$ Advantage ${ }^{\circledR}$ qPCR Premix Kit (TaKaRa, \#639676). The primers used for qRT-PCR are listed in Supplementary Table-2. $\beta$-Actin was set as an internal control. The relative expression levels of genes were calculated using the $2^{-\Delta_{C t}}$ method in which $\Delta C t=C t^{\text {Gene }}-C t^{\text {Actin }}$.

\section{Western blotting analysis}

The immunoblot analysis was performed as described previously [35]. Briefly, tissues and cells were lysed in 1×RIPA buffer (Sigma-Aldrich, \#R0278). Equal amounts of protein in each sample were loaded into a $10 \%$ SDS-PAGE gel. After transferring to a membrane and blocking with $5 \%$ milk, the proteins were probed with the following primary antibodies: anti-CtBP1 (BD Biosciences, San Jose, CA, USA, \#612042), anti-CtBP2 (BD Biosciences, \#612044), anti-CD31 (ThermoFisher Scientific, \#PA5-16301), anti-CD55 (ThermoFisher Scientific, \#PA5-82005), anti-CD68 (ThermoFisher Scientific, \#MA5-13324), anti-GAPDH (Santa Cruz Biotechnology, Dallas, Texas, USA, \#sc-365062), anti-Caspase-1 (Santa Cruz Biotechnology, \#sc-56036), anti-Flag (Sigma-Aldrich, \#SAB4200071), anti-Myc (Abcam, Cambridge, MA, USA, \#ab9106), anti-p300 (Santa Cruz Biotechnology, \#sc-585), anti-c-Jun (Sigma-Aldrich, \#SAB4501606), anti-c-FOS (Sigma-Aldrich, \#F7799), anti-p50 (ThermoFisher Scientific, \#PA1-30409), anti-p65 (ThermoFisher Scientific, \#14-6731-81), anti-IRF2 (Abcam, \#ab3388), anti-STAT4 (Abcam, \#ab68156), anti-NLRP3 (Abcam, \#ab210491), anti-Il-1ß (Abcam, \#ab2105), anti-DNMT1 (Abcam, \#ab13537), and anti-DNMT3A (Abcam, \#ab2850). After probing with secondary antibodies, protein band signals were detected using a Pierce ${ }^{\mathrm{TM}}$ ECL western blotting substrate (ThermoFisher Scientific, \#32106).

\section{Immunohistochemistry (IHC) staining and immunofluorescence (IF) assays}

The IHC staining assay was performed following a standard procedure. Briefly, tissues from OA patients and healthy controls were fixed in $10 \%$ PBS-buffered formalin for $48 \mathrm{~h}$. The resulting tissues were then performed a series of procedures including dehydration, infiltration and embedding in paraffin, section, and re-dehydration. The obtained slides were probed with primary antibodies including anti-CtBP1, anti-CtBP2, anti-CD31, antiCD55, and anti-CD68, respectively, followed by incubation with secondary biotinylated antibodies for $1 \mathrm{~h}$ at room temperature. The mouse and rabbit 
specific HRP/DAB detection kit (Abcam, \#ab64264) was used to determine the signals of each protein. For IF assay, the HOB-OA cells were fixed with $4 \%$ formaldehyde in PBS at room temperature for $20 \mathrm{~min}$. After rinsing five times with PBS buffer, cells were incubated with the primary antibodies including anti-CtBP1, anti-p300 and anti-c-Jun, respectively, followed by incubation with Alexa Fluor 594 (red) (ThermoFisher Scientific, \#A32740) and 488 (green) (ThermoFisher Scientific, \#A32723) conjugated secondary antibodies at room temperature for $1 \mathrm{~h}$. Cells were also stained with the fluorescent 4',6diamidino-2-phenylindole (DAPI) to visualize the nucleus.

\section{Vector construction}

The coding sequences of $C t B P 1, C t B P 2, p 50, p 65$, STAT4, c-Jun, c-FOS, IRF2, p300, DNMT1, and DNMT3A were cloned into the BamHI and EcoRI sites of a pCDNA3-2×Flag vector, respectively. The coding sequences of CtBP1,CtBP2,c-Jun, c-FOS, and p300 were cloned into the BamHI and EcoRI sites of a pCDNA3-6-Myc vector, respectively. The pCDNA3$2 \times$ Flag-p300 vector was used as a template to create

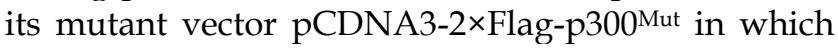
the PMDLS motif was mutated to AMAAS. The promoter of NLRP3 was cloned into the KpnI and Xhol sites of a luciferase vector pGL4.26. The pGL4.26-pNLRP3 vector was used as a template to create its mutant vector pGL4.26-pNLRP3Mut in which the TGAGTCA sequences were mutated to TCCAGCA. All primers used for vector constructions were listed in Supplementary Table-3.

\section{Cell transfection}

Cells were seeded into 6-well plates at a density of $4 \times 10^{5}$ and then further grown for another $30 \mathrm{~h}$ to reach nearly $80 \%$ confluence. Cells were washed twice with PBS buffer and incubated in the fresh chondrocyte growth medium. A total of $1 \mu \mathrm{g}$ plasmid DNA or $20 \mathrm{pM}$ siRNAs was mixed completely with $100 \mu \mathrm{L}$ Opti-MEM (ThermoFisher Scientific, \#31985062). In addition, $5 \mu \mathrm{L}$ Lipofectamine 2000 (ThermoFisher Scientific, \#11668019) was mixed with $95 \mu \mathrm{L}$ Opti-MEM. Then, the DNA-Lipofectamine 2000 complexes were added into each well containing cells and medium. After completely mixing, the cells were incubated at $37^{\circ} \mathrm{C}$ for $48 \mathrm{~h}$ until further experiments. For knockdown analysis of each gene, two independent siRNAs targeting different regions of genes were used. All siRNAs were ordered from Sigma-Aldrich and they included siCtBP1 (\#SASI_Hs01_00243819 and \# SASI_Hs02_00306193), siCtBP2 (\#SASI_Hs02_00317301 and \#SASI_Hs02 00317304), sip50 (\#SASI_Hs02_00336720 and \#SASI_
Hs01_00227679), sip65 (\#SASI_Hs01_00171090 and \#SASI_Hs01_00171091), siSTAT4 (\#SASI_Hs01_ 00175605 and SASI_Hs01_00175608), sic-Jun (\#SASI_ Hs01_00150279 and \#SASI_Hs01_00150282), si-c-FOS (\#SASI_Hs01_00184572 and \# SASI_Hs01_00184573), siIRF2 (\#SASI_Hs02_00333421 and \# SASI_Hs01_ 00204944), sip300 (\#SASI_Hs01_00052827 and \#SASI_ Hs01_00052819), siDNMT1 (\#SASI_Hs01_00204021 and \#SASI_Hs01_00204022), and siDNMT3A (\#SASI_ Hs01_00160193 and \# SASI_Hs01_00160197).

\section{Microarray analysis}

The microarray analysis procedure was the same as a previous protocol [35]. Briefly, $2.0 \mu \mathrm{g}$ total RNA from each sample was labeled with biotin. After cDNA was synthesized using a GeneChip 3' IVT Express Kit (ThermoFisher Scientific, \#902416), the resulting products were fragmented and hybridized with a GeneChip Human Genome U133 Plus 2.0 (ThermoFisher Scientific, \#900466) at $45^{\circ} \mathrm{C}$ for $12 \mathrm{~h}$. The chip was stained with streptavidin-phycoerythrin (SAPE) for 300 seconds at $35^{\circ} \mathrm{C}$, followed by scanning in a GeneArray Scanner to collect data.

\section{Luciferase assay}

The luciferase assay was carried out following a previous protocol [41]. Briefly, the combinations of pGL4.26-pNLRP3 + pRL-TK-Renilla or pGL4.26pNLRP3 $^{\text {Mut }}+$ pRL-TK-Renilla plasmids were transfected into HC-OA-c-Jun-KD (knockdown), HAC-OA-c-FOS-KD, HC-OA-c-Jun-OE (overexpression) and HC-OA-c-FOS-OE cells, respectively. Cells were further cultured at $37^{\circ} \mathrm{C}$ for $48 \mathrm{~h}$, followed by luciferase assays with a Nano-Glo Dual-Luciferase Reporter Assay System (Promega, Madison, WI, USA, \#N1610) according to the manufacturer's protocol.

\section{Immunoprecipitation (IP) and Co-IP analyses}

For IP analyses, the pcDNA3-2×Flag-CtBP1 and pcDNA3-2 $\times$ Flag-CtBP2 plasmids were transfected into HC-OA cells. After incubation for $48 \mathrm{~h}$, cells were lysed with $1 \times$ RIPA buffer supplemented with a protease inhibitor cocktail (Roche, Shanghai, China, \#11697498001). Cell lysates were centrifuged at 14000 rpm for $15 \mathrm{~min}$, followed by incubation with antiFlag-agarose (Sigma-Aldrich, \#A4596) at $4^{\circ} \mathrm{C}$ for $2 \mathrm{~h}$. The Flag beads were rinsed with $1 \times$ RIPA buffer four times. The resulting Flag-CtBP-associated protein complexes were subjected to immunoblot analyses. For Co-IP analysis, the procedures were the same as a previous protocol [35]. Briefly, different combinations of Flag-tagged and Myc-tagged plasmids as shown in figures were co-transfected into cells, respectively. After incubation at $37^{\circ} \mathrm{C}$ for another $48 \mathrm{~h}$, cells were lysed and incubated with anti-Flag agarose beads and 
anti-c-Myc agarose beads (Sigma-Aldrich, \#A7470) at $4^{\circ} \mathrm{C}$ for $2 \mathrm{~h}$. The beads were rinsed with $1 \times$ RIPA buffer four times, and protein levels were examined using western blotting assays.

\section{Cell treatments}

HC-OA cells at less than $80 \%$ confluence were washed twice with PBS buffer and then incubated in fresh medium for $30 \mathrm{~min}$. Cells were then treated with different chemicals including $5 \mathrm{ng} / \mathrm{mL}$ or $50 \mathrm{ng} / \mathrm{mL}$ TNF- $\alpha$ alone, $1 \mathrm{mM}$ MTOB (4-methylthio-2oxobutyric acid) $+5 \mathrm{ng} / \mathrm{mL}$ TNF- $\alpha$ combination, 1 $\mathrm{mM}$ MTOB $+50 \mathrm{ng} / \mathrm{mL}$ TNF- $\alpha$ combination, $5 \mu \mathrm{M}$ or $10 \mu \mathrm{M}$ DNA methylation inhibitor AZA (5-aza-2'-deoxycytidine) (Sigma-Aldrich, \#A3656), $5 \mu \mathrm{M}$ or $\quad 10 \mu \mathrm{M}$ histone deacetylase inhibitor TSA (trichostatin A) (Sigma-Aldrich, \#T8552).

\section{Chromatin immunoprecipitation (ChIP) assay}

ChIP assay was carried out following a previous protocol [35]. Briefly, cells under $80 \%$ confluence were washed twice with PBS and then crosslinked with 1\% formaldehyde in PBS for 15 min. The crosslinked cells were subjected to sonication to break the DNA fragments to an average size of $300-500 \mathrm{bp}$. After centrifugation at $14000 \mathrm{rpm}$ for $15 \mathrm{~min}$, half of the supernatant was used as input, and the other half of supernatant was subjected to ChIP assay with a Millipore ChIP Assay Kit (Millipore, Burlington, MA, USA, \#17295) according to the manufacturer's instructions. The antibodies used in the ChIP assay included anti-CtBP1, anti-CtBP2, anti-p300, anti-cFOS and anti-c-Jun. All of these antibodies were the same as the ones used in the immunoblot analysis. The purified output DNA was subjected to qRT-PCR analyses using primers included in Supplementary Table-4. The relative enrichment was determined using the $2^{-\Delta_{C t}}$ method in which $\Delta C t=C t^{\text {Output }}-C t^{\text {Input }}$.

\section{Enzyme-linked immunosorbent assay (ELISA) assay}

The ELISA assays in the supernatant of cell culture were performed to measure the secreted cytokines including IL-1 $\beta$, IL- 6 , TNF- $\alpha$, and IL-4 following a previous protocol [42]. Briefly, the CtBP1$\mathrm{OE}$ and CtBP2-OE cells were seeded into 6-well plates and cultured at $37^{\circ} \mathrm{C}$ for $48 \mathrm{~h}$. The medium was collected and centrifuged at $1500 \mathrm{rpm}$ for $10 \mathrm{~min}$ and the supernatant was applied to ELISA assays using different kits including IL-1 $\beta$ (Abcam, \#ab214025), IL-6 (Abcam, \#ab46027), TNF- $\alpha$ (Abcam, \#ab46087), and IL-4 (Abcam, \# ab46063) following the protocols provided by the manufacturer.
Quantitative methylation-specific PCR (qMSP) assay

The qMSP assay was carried out as described previously [43]. Genomic DNA was isolated from tissues or cells using a Monarch Genomic DNA Purification Kit (New England Biotechnology, Beijing, China, \#T3010L) following the manufacturer's protocol. A total of $2.0 \mu \mathrm{g}$ genomic DNA from each sample was treated with an EZ DNA MethylationGOLD Kit (Zymo Research, Tustin, CA, USA, \#D5006) in which sodium bisulfite converted unmethylated cytosines but not methylated cytosines to uracil. The modified DNA was subjected to qMSP analysis using a TaqMan ${ }^{\circledR}$ Universal Master Mix II non-UNG Kit (ThermoFisher Scientific, \#4440038) with primers included in Supplementary Table-5.

\section{Statistical analysis}

All experiments in this study were independently repeated in triplicate. The data were analyzed using two-tailed Student's $t$-tests with the SPSS software version 22 and presented by mean \pm SD. The significance levels were set at $P<0.05\left(^{*}\right), P<$ $\left.0.01{ }^{* *}\right)$ and $\left.P<0.001{ }^{* * *}\right)$.

\section{Results}

\section{Both $C t B P I$ and $C t B P 2$ were involved in the pathogenesis of $O A$}

Our recent publication reported that $C t B P 2$ was overexpressed in atrophic nonunion tissues and it repressed the expression of bone development and differentiation genes [35]. To determine if CtBPs are also involved in the pathogenesis of OA, we collected 48 paired cartilage tissues from OA patients and from patients who had no OA symptoms but underwent joint surgeries because of accidents (set as controls) and measured the mRNA levels of $C t B P 1$ and $C t B P 2$ in these biopsies. The qRT-PCR results showed that both CtBP1 and CtBP2 mRNA levels were significantly upregulated nearly 5 -fold in OA tissues compared to controls (Figures 1A and 1B). To further determine if their protein levels were also elevated, we randomly selected three paired biopsies and then examined the protein levels of CtBP1, CtBP2 and three well-known OA markers including CD31 (Cluster of differentiation), CD55 and CD68 [44]. Consistent with their mRNA levels, the western blotting results indicated that both CtBP1 and CtBP2 protein levels were increased $~ 3.5-4$.2-fold (Figures 1C and 1D). The protein levels of three OA markers were also significantly increased $~ 3.5-4.5$-fold in these three OA biopsies (Figures 1C and 1D). Meanwhile, we also performed IHC staining to determine the protein levels of CtBPs and OA markers in both controls and 
OA specimens. Consistent with the immunoblot results, the IHC staining results also indicated that CtBPs and OA markers were significantly induced in OA specimens. Based on these results, we next sought to determine if overexpression of $C t B P s$ could cause the induction of $O A$ markers in vitro. Accordingly, we transfected pCDNA3-2×Flag and pCDNA3-2×Flag$\mathrm{CtBP} 1 / 2$ into HC-OA cells to generate the HC-OA-empty vector (EV) and CtBP overexpression cell lines (CtBP1-OE and CtBP2-OE). After determining $C t B P$ mRNA levels in these cells and HOB-OA cells (Supplementary Figure 1A), we examined the protein levels of three OA markers. As shown in Supplementary Figures $1 B$ and $1 C$, we found that overexpression of $C t B P s$ could induce the protein levels of CD31, CD55 and CD68 up to 3.0-fold. Given that OA markers are routinely activated by inflammation stimuli, we next sought to determine if $C t B P$ overexpression could increase the secretion of proinflammatory cytokines in cell culture. Thus, we measured the concentrations of three proinflammatory cytokines including IL-1 $\beta$, IL-6 and TNF- $\alpha$ and one anti-inflammatory cytokine IL-4 in the supernatant of the medium after culturing CtBP-OE cells. The ELISA results indicated that the secreted
IL-1 $\beta$, IL-6, and TNF- $\alpha$ were significantly increased (Supplementary Figures 1D-1F), while the concentration of IL-4 was not obviously changed in the supernatant of CtBP-OE cells (Supplementary Figure 1G). These results suggested that overexpression of CtBPs might increase proinflammatory cytokine levels and thus activate OA markers.

\section{The expression of NLRP3 was dependent on CtBP levels}

CtBPs function as transcriptional regulators to control gene expression in different biological processes [32]. To determine the genes regulated by CtBPs in the pathogenesis of OA, we conducted a microarray analysis in CtBP-KD and CtBP-OE cells. Accordingly, we created two independent cell lines of CtBP1-KD and CtBP2-KD, respectively, one CtBP1$\mathrm{OE}$ and one CtBP2-OE cell lines. After verifying the successful knockdown and overexpression of $C t B P s$ in these cells (Supplementary Figure 2), we subjected total RNA from HC-OA, CtBP1-KD1, CtBP2-KD1, $\mathrm{CtBP1}-\mathrm{OE}$ and $\mathrm{CtBP2}-\mathrm{OE}$ cells to a microarray analysis. In total, we identified 58 genes controlled by CtBP1 (Supplementary Table-6) and 73 genes

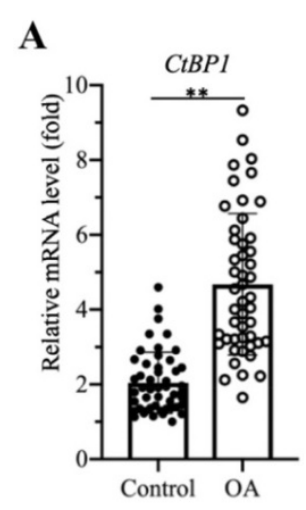

B

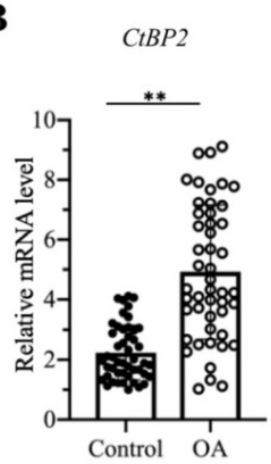

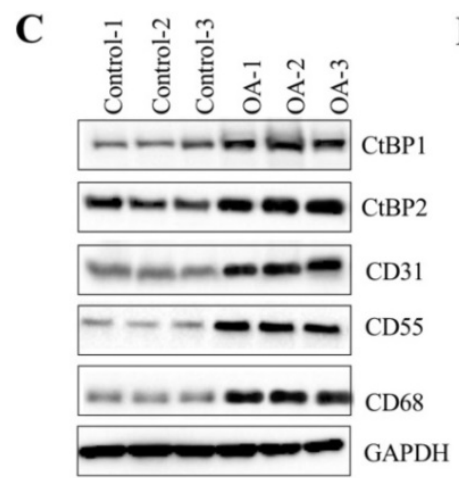

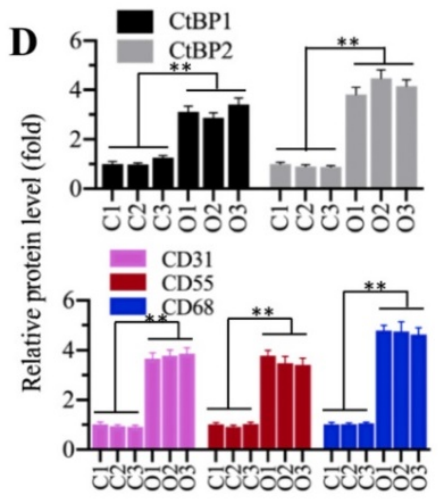

$\mathbf{E}$
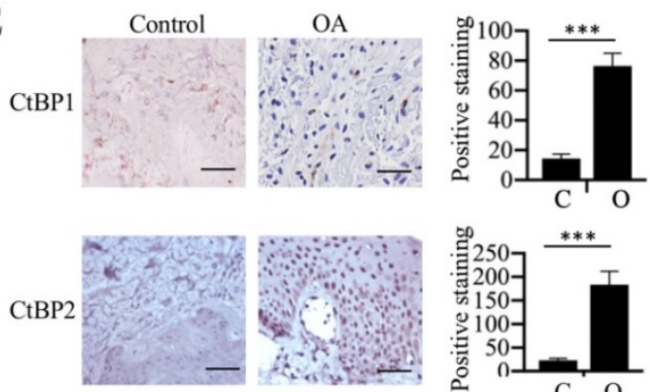

CD31
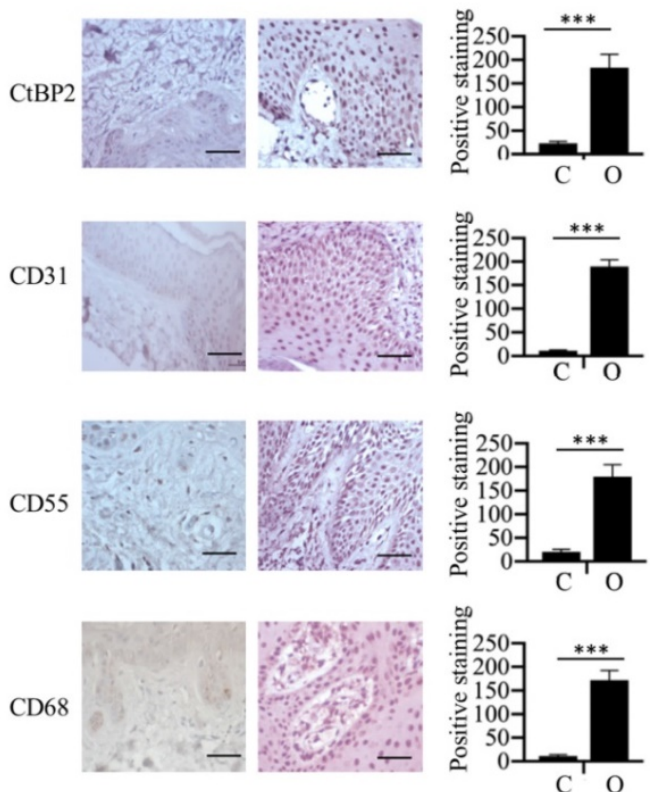

Figure 1. CtBP mRNA and protein levels were significantly increased in OA biopsies. (A and B) The relative $m R N A$ levels of $C t B P 1$ and $C t B P 2$. Total RNA from biopsies of 48 OA patients and 48 controls was used to determine the mRNA levels of CtBP1 (A) and CtBP2 (B) by qRT-PCR analyses. The expression of $\mathrm{CtBP1}$ and $\mathrm{CtBP} 2$ in a healthy control was defined as one-fold. $* * P<0.01$. (C and $\mathbf{D})$ The protein levels of CtBPs and OA markers in three-paired biopsies. Total cell extracts of three-paired biopsies from OA patients and controls were subjected to western blotting to examine the protein levels of CtBP1, CtBP2, CD31, CD55 and CD68 (C). GAPDH was used as a loading control. (D) Statistical data of the relative protein levels of CtBPs and OA markers in (C). $* * P<0.01$. (E) IHC staining results of $\mathrm{CtBPs}$ and OA markers. The same biopsies as $(\mathrm{C})$ were used to IHC staining and representative CtBP1, CtBP2, CD31, CD55 and CD68 images were shown. Bars $=100 \mu \mathrm{m}$. The positive cell numbers were counted and shown in the right panels. C: Control; O: OA. ***P<0.001. 
regulated by CtBP2 (Supplementary Table-7) (Supplementary Figure 3), respectively. Comparing these two gene lists, we surprisingly found that a total of 29 overlapping genes (Supplementary Table-6 and -7). As shown in Figure 2A, we generated a heatmap of 20 genes that were both dependent on CtBP1 and $C t B P 2$. Of these genes, we found several important genes including NLRP3, which is a critical member of the inflammasome, $I L-1 B$ and $I L-6$, two critical proinflammatory cytokines. These results further supported our above hypothesis in which CtBPs were involved in the pathogenesis of OA by increasing proinflammatory cytokines and thus activating OA markers. To test the accuracy of our microarray results, we randomly selected 6 genes including NLRP3, IL-1B, S100A8, Bax, Bim and CDH1 and examined their expression in CtBP-KD and CtBP-OE cells. Consistent with the microarray results, we found that the expression of NLRP3, IL-1B and S100A8 was increased with $C t B P$ overexpression but decreased with $C t B P$ knockdown (Figures 2B-2D). Conversely, the expression of $\mathrm{Bax}, \mathrm{Bim}$ and $\mathrm{CDH} 1$ was decreased with $C t B P$ overexpression but increased with $C t B P$ knockdown (Figures $2 \mathrm{E}-2 \mathrm{G}$ ).

\section{Overexpression of $\mathrm{CtBPs}$ caused the activation of NLRP3 downstream signaling}

Since we observed overexpression of CtBPs in OA biopsies and found that the expression of NLRP3 was dependent on $C t B P$ levels, we next sought to examine the mRNA level of NLRP3 in OA biopsies.
The qRT-PCR results showed that the average NLRP3 mRNA level was upregulated $\sim 5$-fold in OA tissues compared to controls (Figure 3A). It is well known that the NLRP3-associated inflammasome can activate Caspase-1, which subsequently leads to the processing of IL-1 $\beta$ [45]. Thus, the significant increase in NLRP3 in OA biopsies would also activate its downstream signaling. For this purpose, we examined NLRP3, Caspase- 1 and IL-1 $\beta$ levels in three-paired tissues from OA patients and controls. As expected, the western blotting results showed that all these three proteins were activated in OA tissues but not in controls (Figure 3B and Supplementary Figure 4A). Meanwhile, we also detected CtBPs and OA makers in these tissues. The immunoblot results consistently showed that both CtBPs and OA makers were induced in $\mathrm{OA}$ tissues compared to controls (Figure 3B and Supplementary Figure 4A). To further evaluate whether the activation of NLRP3 and Caspase-1 was dependent on CtBPs, we also determined NLRP3, Caspase-1 and IL-1 $\beta$ levels in CtBP-KD and CtBP-OE cells. The results indicated that both NLRP3, cleaved Caspase- 1 and IL-1 $\beta$ protein levels were increased in CtBP-OE cells but were decreased in CtBP-KD cells (Figures 3C, 3D and Supplementary Figures $4 \mathrm{~B}$ and $4 \mathrm{C}$ ). Interestingly, the similar expression patterns in OA markers were also observed in these cells (Figures 3C, 3D and Supplementary Figures 4B and 4C).
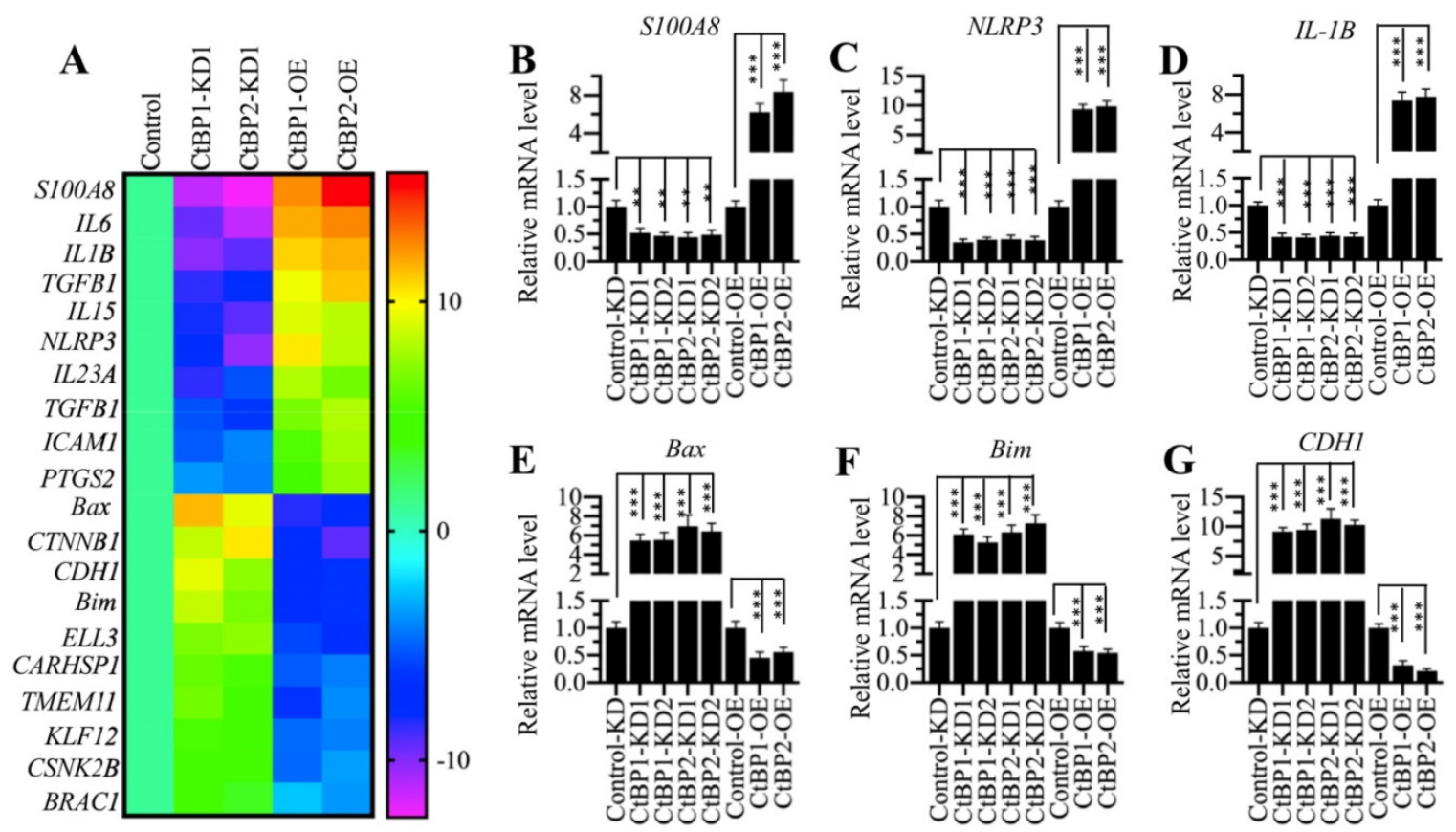

Figure 2. The identification of differentially expressed genes dependent on CtBPs. (A) Heatmaps of genes coregulated by $C t B P I$ and $C t B P 2$. Total RNA from HC-OA (control), CtBPI-KDI, CtBP2-KD1, CtBPI-OE and CtBP2-OE cells were subjected to a microarray analysis. Genes coregulated by $C t B P 1$ and $C t B P 2$ were shown. (B-G) Examination of the expression levels of genes identified in the microarray analysis. Six genes including SIO0A8 (B), NLRP3 (C), ILIB (D), Bax, (E) $\mathrm{Bim}(\mathbf{F})$, and $\mathrm{CDHI}(\mathbf{G})$ were selected to verify their expression in HC-OA-siControl (Control-KD), CtBPI-KD1, CtBP1-KD2, CtBP2-KDI, CtBP2-KD2, HC-OA-PCDNA3-2×Flag (Control-OE), CtBPI-OE and CtBP2-OE cells. The expression of these genes in controls was defined as one-fold. $* * P<0.01$ and $* * * P<0.001$. 
A

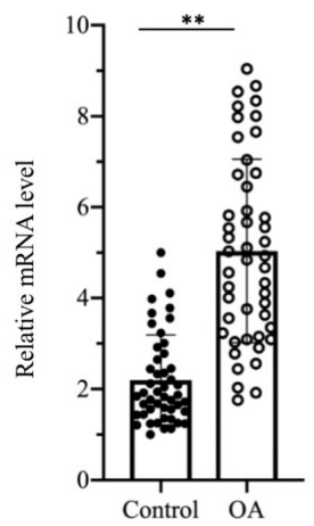

B

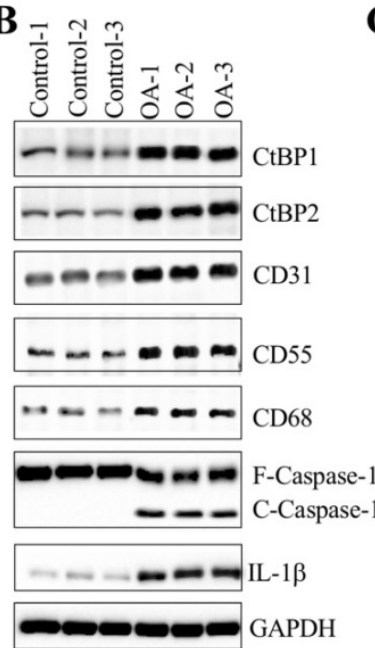

C

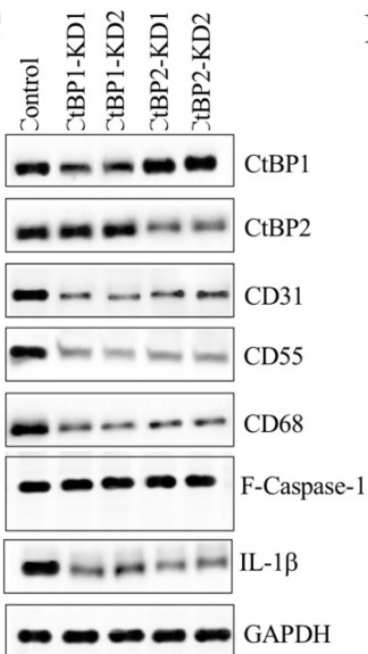

D

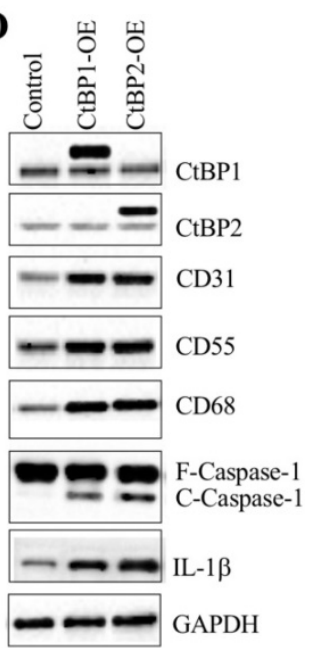

Figure 3. NLRP3 and its downstream signaling were dependent on CtBPs. (A) The relative mRNA levels of NLRP3. The mRNA level of NLRP3 was measured in 48-paired biopsies from OA patients and controls by qRT-PCR analyses. The expression of NLRP3 in a healthy control was defined as one-fold. **P<0.01. (B) NLRP3 and its downstream signaling were activated in OA biopsies. Total cell extracts of three-paired biopsies from OA patients and controls were subjected to western blotting to examine the protein levels of CtBP1, CtBP2, CD31, CD55, CD68, NLRP3, Caspase-1 and IL-1 1 . GAPDH was used as a loading control. (C) The downregulation of $C_{t B P s}$ inactivated NLRP3 and its downstream signaling in vitro. Total proteins isolated from Control-KD, CtBPI-KDI, CtBP1-KD2, CtBP2-KD1 and CtBP2-KD2 cells were used for western blotting analyses to measure the protein levels of CtBP1, CtBP2, CD31, CD55, CD68, NLRP3, Caspase-1 and IL-1 $\beta$. GAPDH was used as a loading control. (D) Overexpression of CtBPs activated NLRP3 and its downstream signaling in vitro. Total proteins isolated from Control-OE, CtBP1-OE and CtBP2-OE cells were used for western blotting analyses to measure the protein levels of CtBP1, CtBP2, CD31, CD55, CD68, NLRP3, Caspase-1 and IL-1ß. GAPDH was used as a loading control.

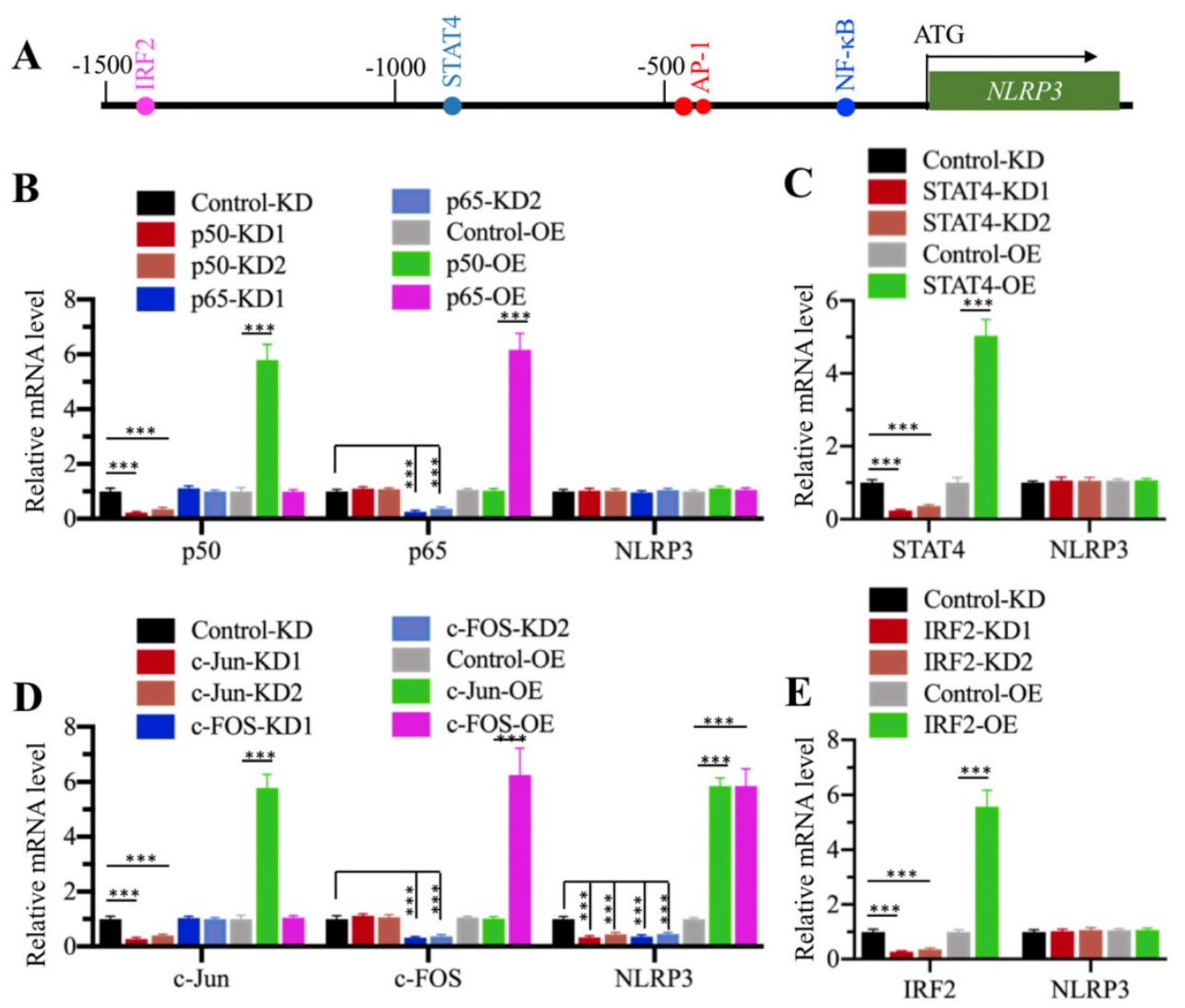

Figure 4. AP1 specifically regulated the expression of NLRP3. (A) Transcription factor binding sites in the promoter of NLRP3. A 1500-bp region in the NLRP3 promoter was used to analyze transcription factor binding sites, and one NF-KB, two API, one STAT4 and one IRF4 binding sites were found. Their binding positions were indicated. (B) Effects of knockdown and overexpression of NF- $k B$ subunits on NLRP3 expression. The Control-KD, p50-KDI, p50-KD2, p65-KDI, p65-KD2, Control-OE, p50-OE and $p 65-\mathrm{OE}$ cells were used to examine the mRNA levels of $p 50, p 65$ and NLRP3. The expression of these genes in controls was defined as one-fold. ***P<0.001. (C) Effects of knockdown and overexpression of STAT4 on NLRP3 expression. The Control-KD, STAT4-KD1, STAT4-KD2, Control-OE and STAT4-OE cells were used to examine the mRNA levels of STAT4 and NLRP3. The expression of these genes in controls was defined as one-fold. ****P<0.001. (D) Effects of knockdown and overexpression of AP-1 subunits on NLRP3 expression. The Control-KD, c-Jun-KD1, c-Jun-KD2, c-FOS-KD1, c-FOS-KD2, Control-OE, c-Jun-OE and c-FOS-OE cells were used to examine the mRNA levels of c-Jun, c-FOS and NLRP3. The expression of these genes in controls was defined as one-fold. $* * * P<0.001$. (E) Effects of knockdown and overexpression of IRF2 on NLRP3 expression. The Control-KD, IRF2-KD1, IRF2-KD2, Control-OE and IRF2-OE cells were used to examine the mRNA levels of IRF2 and NLRP3. The expression of these genes in controls was defined as one-fold. ***P<0.001. 


\section{Transcription factor API specifically regulated the expression of $N L R P 3$ in vitro}

CtBPs only function as transcriptional coregulators, and they need to associate with other proteins to form a transcriptional complex [32]. To determine the transcription factor that regulates NLRP3 expression, we primarily analyzed the transcription factor binding sites in a 1500-bp region of the NLRP3 promoter. We found one NF- $\mathrm{KB}$ site [GGGGTGCCTC, -179-(-)188], two AP1 sites [TGAGTCA, -470-(-476) and -477-(-)483], one STAT4 site [AATTCC, -838-(-)844], and one IRF2 (Interferon regulatory factor 2) site [AAGTGA, -1422-(-)1428] (Figure 4A). To determine if any of these transcription factors could directly regulate the expression of NLRP3, we individually knocked down and overexpressed these transcription factors in HC-OA cells and then examined NLRP3 mRNA levels. After knockdown or overexpression of two NF-kB subunits including $p 65$ and $p 50$, we did not observe a significant change in NLRP3 mRNA level (Figure 4B and Supplementary Figures 5A and 5B). Similarly, we also did not identify a significant change in NLRP3 mRNA level in STAT4-KD and STAT4-OE cells (Figure 4C and Supplementary Figures 5C and 5D). However, NLRP3 mRNA level was significantly decreased when AP1 subunits (c-Jun and c-FOS) were knocked down and dramatically increased when AP1 subunits were overexpressed (Figure 4D and Supplementary Figures 5E and 5F). In addition, we also found that knockdown or overexpression of IRF2 did not affect NLRP3 mRNA level (Figure 4E and Supplementary Figures $5 \mathrm{G}$ and $5 \mathrm{H}$ ). These results suggest that AP1 subunits could specifically regulate the expression of NLRP3 in vitro. To further determine if AP1 subunits bound to the promoter of NLRP3 through the consensus sequence TGAGTCA, we constructed a mutant in which both two AP1 sites located in -470-(-476) and -477-(-)483 were changed to TCCAGCA. We co-transfected pGL4.26-pNLRP3 ${ }^{W T}+$ Renilla or pGL4.26-pNLRP3Mut + Renilla into c-Jun-KD, c-FOS-KD, c-Jun-OE, and c-FOS-OE cells, respectively. After determining the expression of c-Jun and c-FOS in these cells (Supplementary Figures $6 \mathrm{~A}$ and 6B), we performed a luciferase assay to compare the changes in luciferase activities. In cells expressing pGL4.26-pNLRP3WT + Renilla, knockdown of either c-Jun or c-FOS could significantly decrease the luciferase activities, while overexpression of either c-Jun or c-FOS markedly induced the luciferase activities (Supplementary Figures 6C and 6D). In contrast, we found that knockdown or overexpression of $c$-Jun or c-FOS could not significantly change the luciferase activities in cells expressing pGL4.26-
pNLRP3Mut + Renilla (Supplementary Figures 6C and 6D). These results clearly suggested that AP1 subunits bound to the promoter of NLRP3 through the consensus sequence TGAGTCA.

\section{CtBPs, p300 and AP 1 subunits assembled a transcriptional complex}

Since both CtBPs and AP1 could activate the expression of NLRP3, we next evaluated whether they could directly form a transcriptional complex. Based on the notion that CtBPs interact with other proteins through a conserved PXDLS motif [32], we primarily analyzed the amino acid sequences of c-Jun and c-FOS to search if they contained this conserved motif or similar motifs, such as PXDLX, XXDLS and PXDXS. Unfortunately, we were unable to identify any of these motifs (Supplementary Figure 7). Based on the notion that transcription factors commonly interact with HATs such as p300 and CBP to activate gene expression, and CtBPs have been shown to directly interact with p300 through the PXDLS motif [28, 32], we proposed that $\mathrm{p} 300$ could function as a linker to directly interact with both $\mathrm{CtBPs}$ and AP1, thus forming a complex. To verify this hypothesis, we performed IP assays using CtBP1-OE and CtBP2-OE cells in the HC-OA background. After purification, the Flag-CtBP1- and Flag-CtBP2-associated protein complexes were subjected to western blotting analyses to detect if they could pull down p300 and AP1 subunits. As expected, our results indicated that both CtBP1 and CtBP2 could pull down p300, c-Jun and c-FOS (Figure 5A), which suggested that they could assemble a CtBP-p300-AP1 complex (CPAC). Moreover, $\mathrm{CtBP} 1$ and $\mathrm{CtBP2}$ could also pull down themselves and each other (Figure 5A), which suggested that they might form a heterotetramer. To determine the direct interactions between CtBPs and p300, CtBPs and AP1 subunits, p300 and AP1 subunits, as well as CtBP1 and CtBP2, we constructed the Flag- and Myc-tagged vectors of these proteins and then performed Co-IP assays to examine how the CPAC transcriptional machinery was assembled. The western blotting results showed that CtBPs could directly interact with p300 instead of AP1 subunits (Figure 5B). Moreover, CtBP1 and CtBP2 could also interact with themselves and each other in vitro (Figure 5C). The AP1 subunits could directly interact with p300 (Figure 5D). These results suggested that p300 functioned as a linker to interact with CtBPs and AP1 subunits, thereby assembling the CPAC transcriptional machinery (Figure 5E). To further verify the formation of CPAC, we overexpressed CtBPs in p300-KD cells and then performed IP analyses to determine if the output of AP1 subunits was changed. As shown in Supplementary Figure 8A, 
equal amounts of Flag-CtBPs pulled down much less p300 and AP1 subunits in the p300-KD background cells compared to the HC-OA background cells. To determine if CtBPs interacted with p300 through the PXDLS motif, we created a p300 mutant in which the PMDLS motif was mutated to AMAAS and then examined the direct interactions between CtBPs and p300 ${ }^{\text {Mut, }}$ and AP1 subunits and p300 Mut. The immunoblot results indicated that CtBPs could not interact with $\mathrm{p} 300^{\mathrm{Mut}}$, while the interaction between AP1 subunits and p300Mut was not changed in comparison to the wild type p300 (Supplementary Figure 8B). These results suggested that p300 interacted with CtBPs and AP1 subunits through different domains. In addition, we also carried out IF analyses to determine if CtBPs, p300 and AP1 subunits were colocalized in the nucleus. Accordingly, we examined the colocalizations of CtBP1 and p300, p300 and c-Jun, and CtBP1 and c-Jun. Our results indicated that these three paired proteins were colocalized in the nucleus (Supplementary Figure 8C).
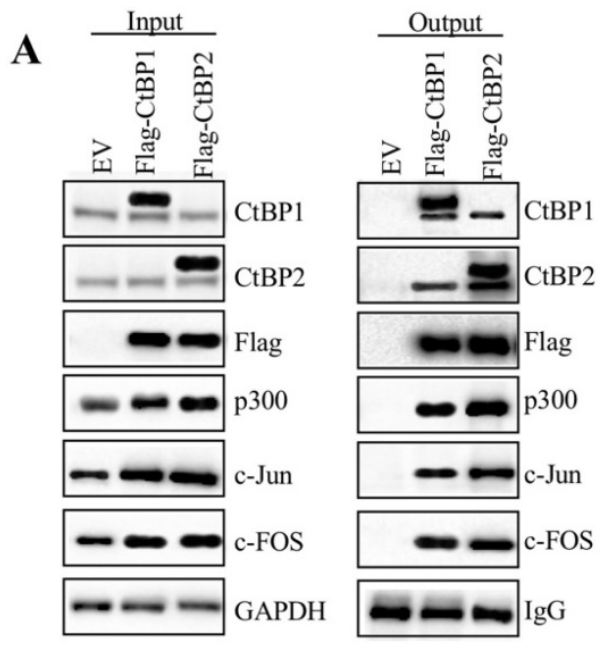

C

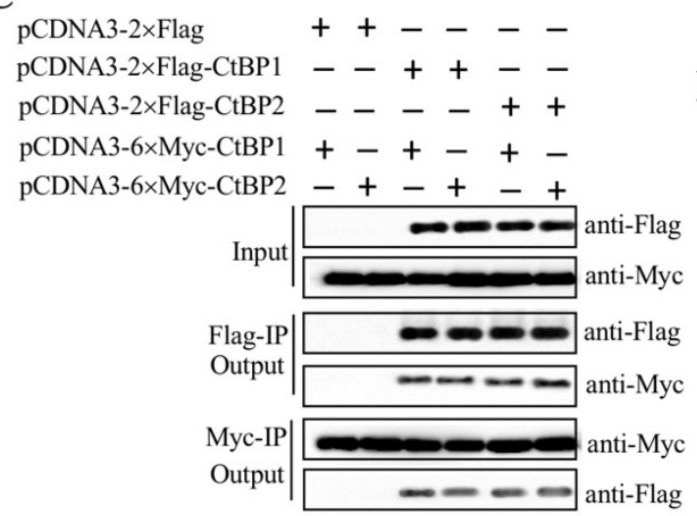

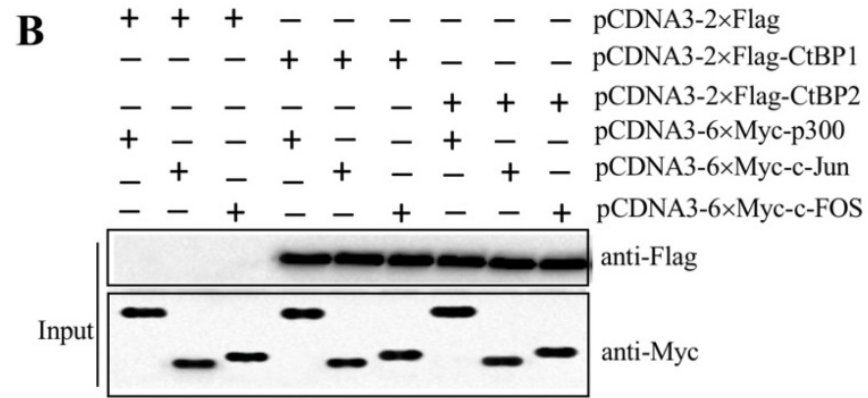
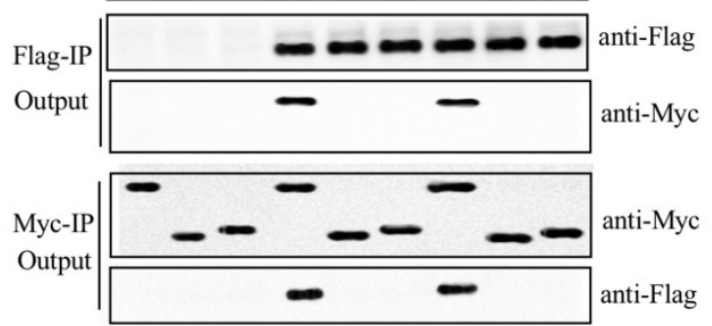

D

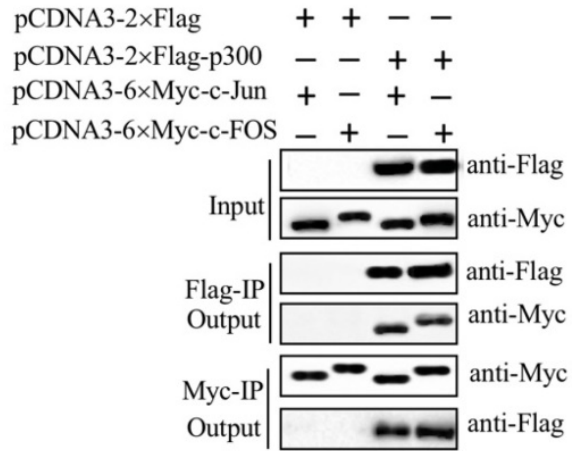

$\mathbf{E}$

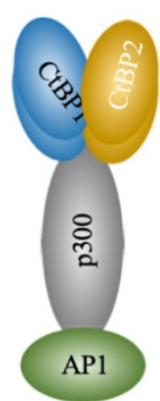

Figure 5. AP1 recruited $\mathrm{p} 300$ and $C t B P s$ to form a transcriptional complex in vitro. (A) Both $C t B P 1$ and $C t B P 2$ associated with $p 300$ and $A P 1$. HC-OA

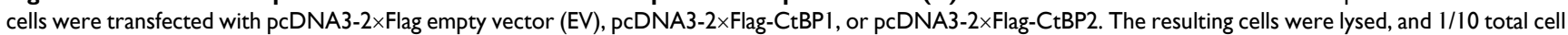
extracts were taken out as input, and the other $9 / 10$ cell extracts were subjected to IP analysis with an anti-Flag agarose. The input (left panel) and output (right panel) proteins were subjected to western blotting to examine protein levels using anti-Flag, anti-CtBPI, anti-CtBP2, anti-p300, anti-c-Jun and anti-c-FOS antibodies, respectively. GAPDH and IgG were used as the loading control of input and output proteins, respectively. (B) CtBPs directly interacted with p300 instead of API

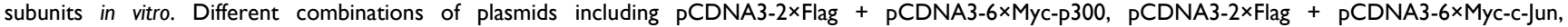

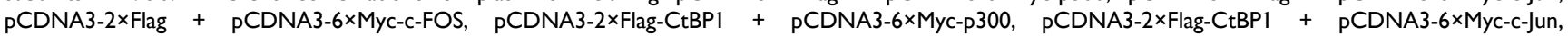

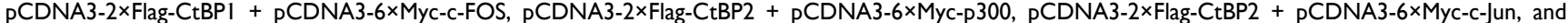
PCDNA3-2×Flag-CtBP2 + pCDNA3-6×Myc-c-FOS were cotransfected into HC-OA cells. After incubating for another 48 h, cells were lysed and $1 / 10$ total cell extracts were taken out as input, and the other $9 / 10$ cell extracts were subjected to IP analysis with an anti-Flag agarose and anti-Myc agarose, respectively. The input and output proteins were probed with anti-Flag and anti-Myc antibodies, respectively. (C) CtBPs formed a heterotetramer in vitro. Different combinations of plasmids

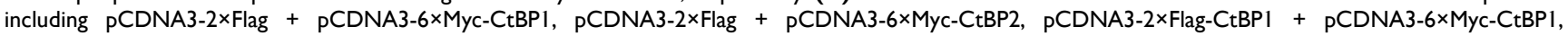

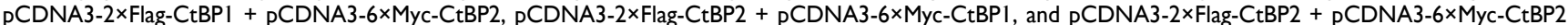
were cotransfected into HC-OA cells. After incubating for another $48 \mathrm{~h}$, cells were lysed and $1 / 10$ total cell extracts were taken out as input, and the other $9 / 10$ cell extracts were subjected to IP analysis with an anti-Flag agarose and anti-Myc agarose, respectively. The input and output proteins were probed with anti-Flag and anti-Myc antibodies, respectively. (D) p300 directly interacted with API subunits in vitro. Different combinations of plasmids including pCDNA3-2×Flag +

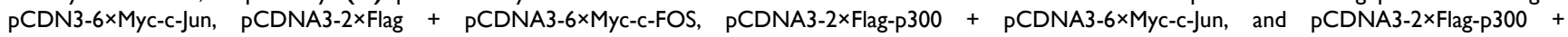
PCDNA3-6 $\times$ Myc-c-FOS were cotransfected into HC-OA cells. After incubating for another $48 \mathrm{~h}$, cells were lysed and $1 / 10$ total cell extracts were taken out as input, and the other $9 / 10$ cell extracts were subjected to IP analysis with an anti-Flag agarose and anti-Myc agarose, respectively. The input and output proteins were probed with anti-Flag and anti-Myc antibodies, respectively. (E) A schematic model of the CPAC transcriptional machinery. API subunits directly interacted with p300, which recruited the CtBP heterotetramer to assemble a complex. 


\section{Knockdown of CtBPs, $p 300$ or API subunits decreased their bindings to the promoter of NLRP3}

Our above results indicated that CtBPs and AP1 were required for NLRP3 transactivation, we next also aimed to evaluate whether p300 had a similar effect. Consequently, we knocked down and overexpressed p300 in HC-OA cells and examined the mRNA levels of NLRP3. The qRT-PCR results indicated that knockdown of $p 300$ also caused a decrease in NLRP3 mRNA level, while overexpression of $p 300$ resulted in its induction (Supplementary Figure 9A). We next sought to determine if the CPAC was able to directly bind to the NLRP3 promoter. For this purpose, we performed ChIP assays in CtBP-OE and CtBP-KD cells using anti-CtBP1, anti-CtBP2, anti-p300, anti-c-Jun and anti-c-FOS antibodies. Our results showed that the enrichment of the individual members of CPAC in the NLRP3 promoter was significantly increased in CtBP1-OE cells but dramatically decreased in CtBP-KD cells (Supplementary Figures 9B and 9C). In addition, we also performed ChIP assays in p300-KD, p300-OE, c-Jun-KD, c-Jun-OE, c-FOS-KD and c-FOS-OE cells using anti-CtBP1, anti-CtBP2, anti-p300, anti-c-Jun and anti-c-FOS antibodies. Similarly, we also observed that the enrichment of CPAC members in the NLRP3 promoter was significantly increased in the overexpressed cells but dramatically decreased in the knockdown cells (Supplementary Figures 10A and 10B). These results clearly indicated that the CPAC transcriptional machinery specifically bound to the promoter of NLRP3 and activated its expression.

\section{Inhibition of CtBPs with the specific inhibitor MTOB impaired NLRP3 expression}

Since CtBPs functioned as activators to regulate NLRP3 expression, we speculated that the blockage of CtBP function should inhibit NLRP3 expression. For this purpose, we treated HC-OA and HOB-OA cells with the proinflammatory cytokine TNF- $\alpha$ to mimic inflammation in vitro. Our results showed that $C t B P 2$, p300, c-Jun, c-FOS and NLRP3 mRNA levels were upregulated with TNF- $\alpha$ treatment in a dosedependent manner (Figures 6A and 6B). However, we only observed a slight induction of CtBP1 mRNA level in HOB-OA cells but not in HC-OA cells with TNF- $\alpha$ treatment (Figures $6 \mathrm{~A}$ and $6 \mathrm{~B}$ ). Next, we treated HC-OA and HOB-OA cells with the CtBP inhibitor MTOB prior to TNF- $\alpha$ stimulation and measured the expression of CtBPs and NLRP3. As shown in Figures $6 \mathrm{C}$ and $6 \mathrm{D}$, inhibition of CtBP functions did not change the mRNA levels of $C t B P 1$ and $C t B P 2$ but significantly decreased the expression of NLRP3. To further evaluate whether MTOB treatment could decrease the binding of CPAC members to the promoter of NLRP3, we carried out ChIP assays in cells treated with both MTOB and TNF- $\alpha$ using anti-CtBP1, anti-CtBP2, anti-p300, anti-c-Jun and anti-c-FOS antibodies. Our results indicated that the enrichment of CPAC members in the NLRP3 promoter was significantly decreased in cells treated with MTOB+TNF- $\alpha$ compared to cells only treated with TNF- $\alpha$ (Figure $6 \mathrm{E}$ ). These results suggested that the blockage of CtBPs with the specific inhibitor MTOB impaired the binding of CPAC to the promoter of NLRP3 and thus inhibited its expression.

\section{Decreased DNA methylation levels in the promoters of CtBPs caused their overexpression in OA biopsies}

The overexpression of $C t B P$ mRNAs in OA biopsies suggested that they were regulated at the transcriptional level. To reveal the underlying mechanism of $C t B P$ overexpression, we analyzed their promoters (a 1500-bp region upstream of the ATG) in a database (http:/ / www.urogene.org) and found that the promoters of $C t B P 1$ and $C t B P 2$ contained two and three CpG islands, respectively (Figures 7A and 7B). These results suggested that DNA methylation might be involved in the regulation of $C t B P$ overexpression. Following this, we treated genomic DNA from 48 OA patients and controls with sodium bisulfite and then examined DNA methylation levels using primers specifically located in each CpG island. The qMSP results showed that DNA methylation levels in all CpG islands were significantly decreased in DNA from OA patients compared to controls (Figures 7C-7G). Given that DNA methylation is mainly mediated by DNMT1 (DNA methyltransferase 1) and DNMT3, we also examined both DNMT1 and DNMT3A mRNA levels in biopsies of OA patients and controls. Our results indicated that both DNMT1 and DNMT3A mRNA levels were slightly downregulated in OA patients compared to controls (Supplementary Figures 11A and 11B). To determine if knockdown and overexpression of DNMTs could change the expression of CtBPS and NLRP3, we generated DNMT1-KD, DNMT1-OE, DNMT3A-KD and DNMT3A-OE cell lines (Supplementary Figure 11C), followed by measuring mRNA and protein levels of CtBPs and NLRP3. Our results indicated that the mRNA and protein levels of CtBPs and NLRP3 were increased in DNMT1-KD and DNMT3A-KD cells but decreased in DNMT1-OE and DNMT3A-OE cells (Supplementary Figures 11D-11G). In addition, we also treated HC-OA cells with the DNA methylation inhibitor AZA and then measured CtBPs and NLRP3 mRNA levels. Our results showed that 
both CtBPs and NLRP3 mRNA levels were significantly increased with AZA treatment in a dose-dependent manner (Supplementary Figures 12A and 12B). In contrast, treatments with the histone deacetylase inhibitor TSA did not affect the expression of CtBPS and NLRP3 (Supplementary Figures $12 \mathrm{~A}$ and $12 \mathrm{~B}$ ). We also observed the similar effects of AZA and TSA treatments on the protein levels of CtBPs and NLRP3 (Supplementary Figures $12 \mathrm{C}$ and 12D). In addition, we also performed ChIP assays to determine the enrichment of CPAC members in the promoter of NLRP3 in cells treated with AZA and in cells overexpressing or knocking down DNMTs. The ChIP assay results indicated that AZA treatment and knockdown of DNMT1 or DNMT3A increased the enrichment of CPAC members in the promoter of NLRP3 (Supplementary Figure 13), while overexpression of DNMT1 or DNMT3A resulted in a decrease in the enrichment of CPAC members in the promoter of NLRP3 (Supplementary Figure 13). These results clearly suggested that the decreased DNA methylation levels were responsible for the overexpression of $C t B P s$ and the activation of CtBP-dependent downstream signaling.
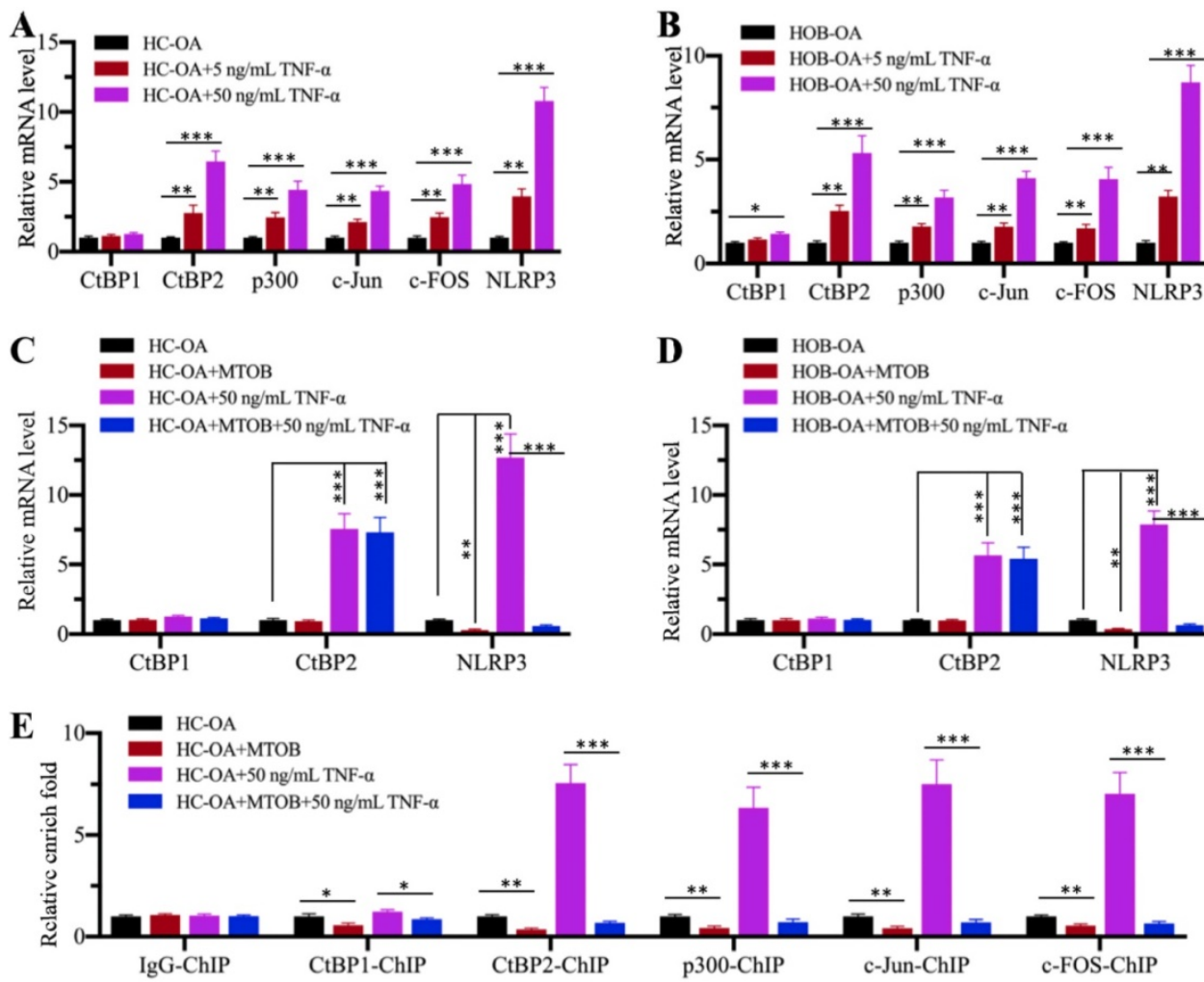

Figure 6. TNF- $\alpha$ activated the CPAC in vitro and the blockage of CtBPs inhibited NLRP3 expression. (A and B) Effects of TNF- $\alpha$ on the expression of CPAC members and NLRP3. HC-OA (A) and HOB-OA (B) cells were treated with different concentrations of TNF- $\alpha$ including 0,5 and $50 \mathrm{ng} / \mathrm{mL}$ for $6 \mathrm{~h}$, followed by RNA isolation and qRT-PCR analyses to examine the mRNA levels of CtBPI, CtBP2, p300, c-jun, c-FOS and NLRP3. **P<0.01 and ***P<0.001. (C and D) Effects of CtBP inhibition on the expression of NLRP3. HC-OA (C) and HOB-OA (D) cells were treated with $1 \mathrm{mM}$ MTOB or $50 \mathrm{ng} / \mathrm{mL}$ TNF- $\alpha$ alone or together for $6 \mathrm{~h}$. The resulting cells were subjected to RNA isolation and qRT-PCR analyses to examine the mRNA levels of CtBPI, CtBP2 and NLRP3. ${ }^{* * P}<0.01$ and $* * * P<0.001$. (E) Effects of $C t B P$ inhibition on the enrichment of CPAC members in the promoter of NLRP3. Cells used in (C) were subjected to ChIP assays with lgG, anti-CtBPI, anti-CtBP2, anti-p300, anti-c-Jun or anti-c-FOS antibody, followed by qRT-PCR analyses to determine the enrichment of these proteins in the NLRP3 promoter. The enrichment of CPAC members in untreated HC-OA cells was defined as one-fold. $* P<0.05, * * P<0.01$ and $* * * P<0.001$. 
A

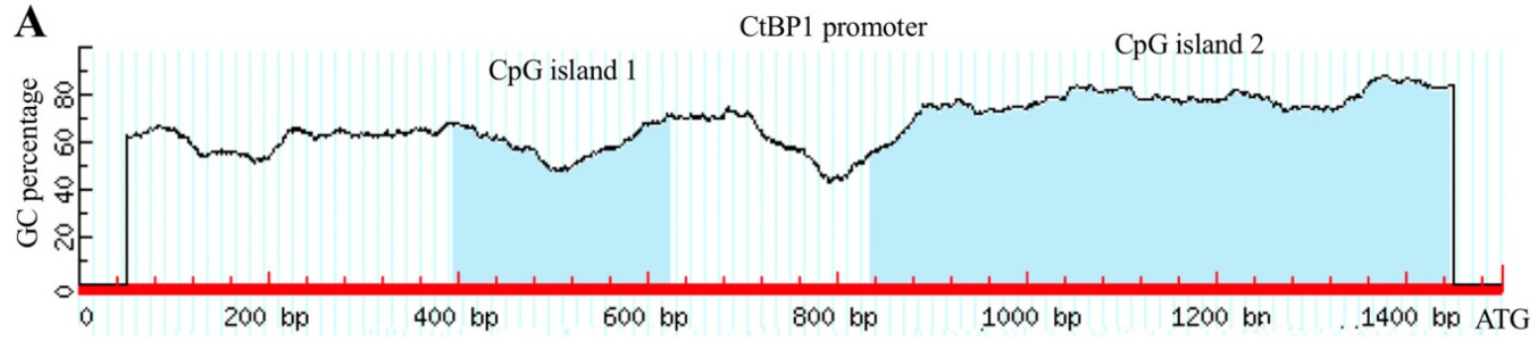

B
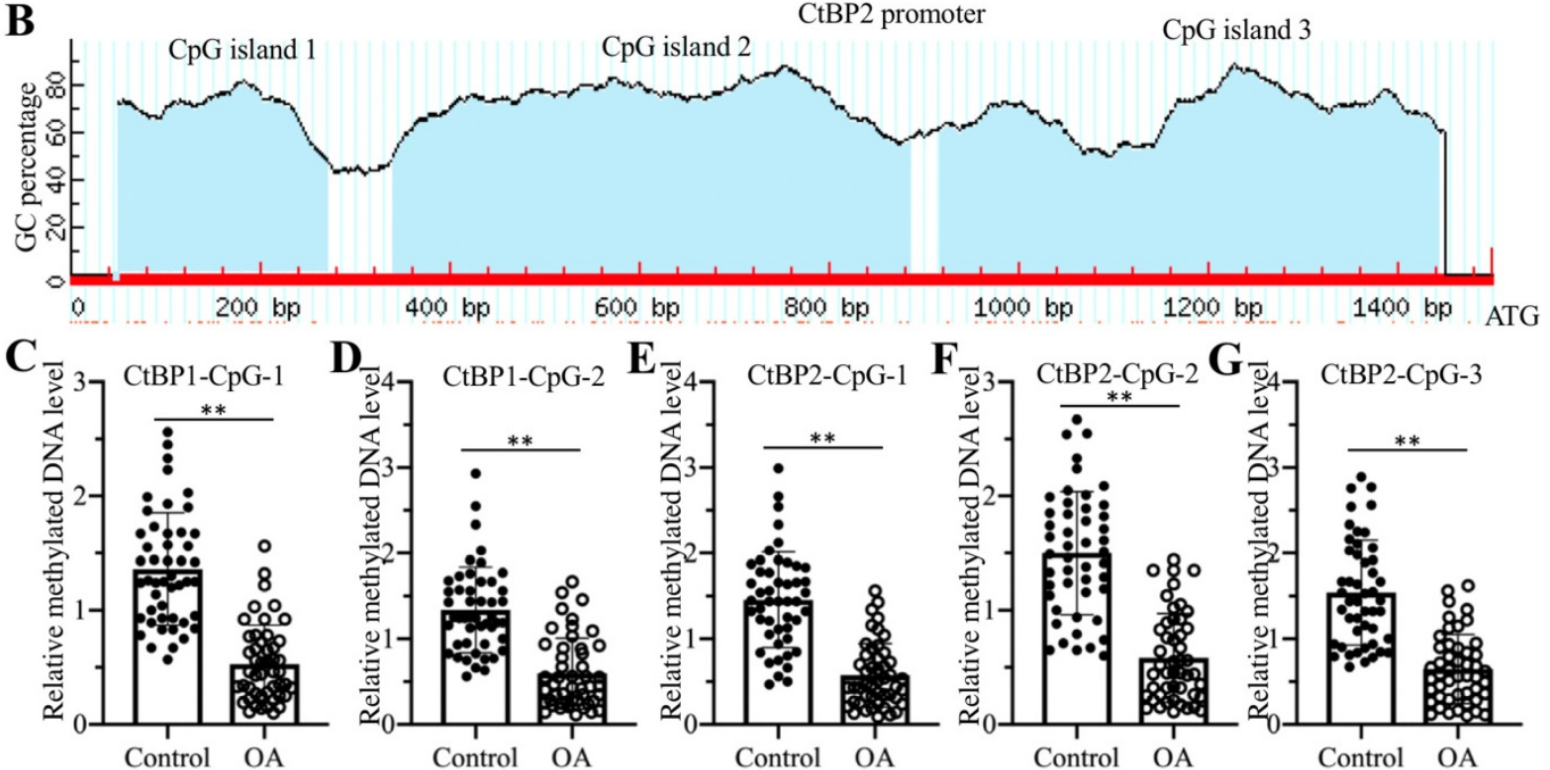

Figure 7. DNA methylation levels in the promoters of $C t B P s$ were decreased in $O A$ biopsies. $(A$ and $B)$ The promoters of $C t B P s$ were abundant in $C_{p} G$ islands. A 1500-bp region in the promoter of either CtBPI or CtBP2 was used to analyze GC contents, and two $C_{p} G$ islands in the promoter of $C t B P I$ (A) and three $C_{p G}$ islands in the promoter of $C_{t B P 2}$ (B) were identified. (C-G) The DNA methylation levels of $C t B P$ promoters were decreased in OA biopsies. Genomic DNA of 48-paired biopsies from OA patients and controls were treated with sodium bisulfite, followed by qMSP analyses to measure methylated DNA levels in the CPG islands of CtBPI (C and $\mathbf{D})$ and $C t B P 2(\mathbf{E}-\mathbf{F})$ promoters. $* * P<0.01$.

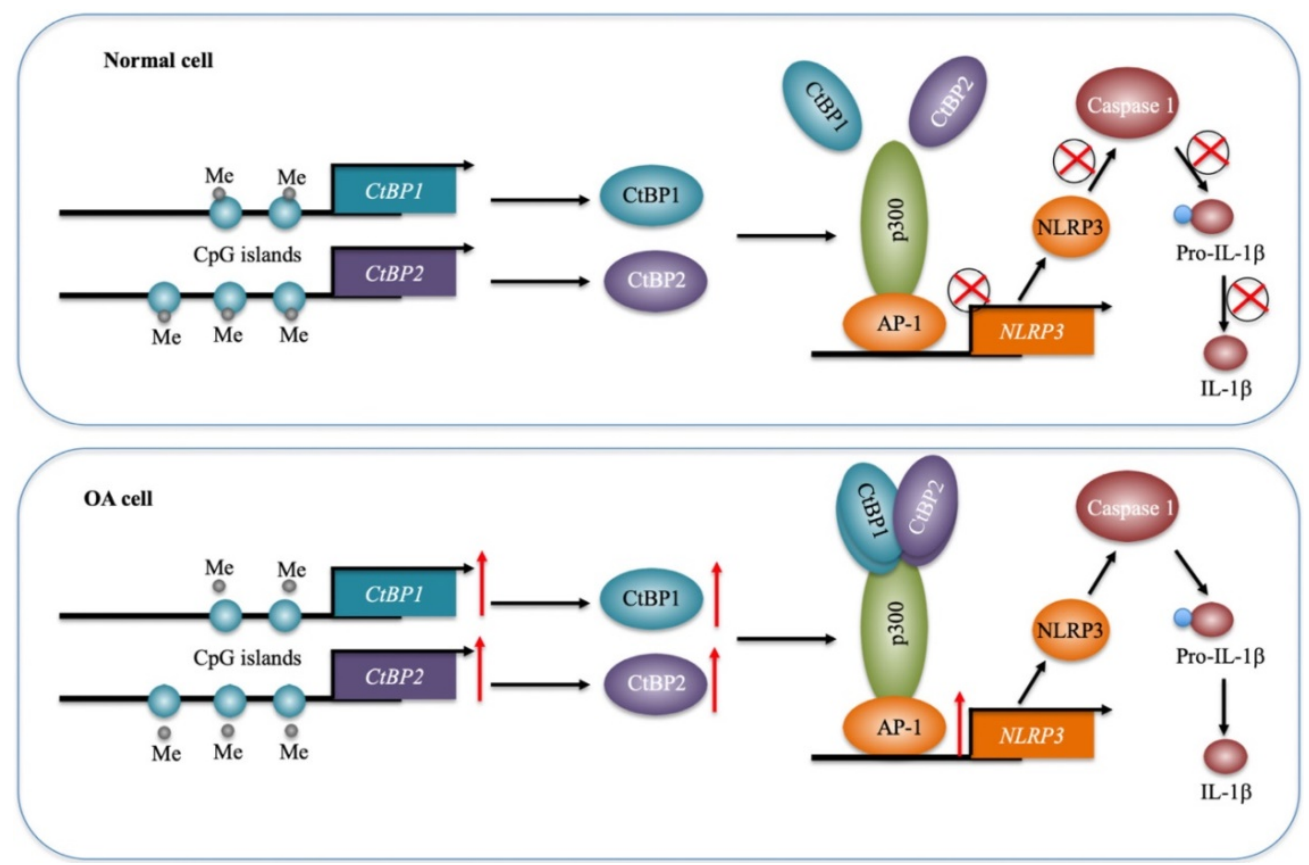

Figure 8. A schematic model of the CPAC machinery in the pathogenesis of OA. In normal cells, DNMTs maintain the DNA methylation of CtBP promoters at a basal level. In this state, the expression of CtBPs is very low. Thus, they cannot efficiently associate with p300 and API subunits to assemble the CPAC machinery, and thus maintaining NLRP3 expression at a basal level. In OA cells, the decreased DNA methylation levels in the promoters of CtBPs mediate their overexpression. Overexpressed CtBPs associate with $\mathrm{p} 300$ and API subunits to assemble the CPAC machinery, which binds to the promoter NLRP3 and activates its expression. The induced NLRP3 further activates Caspase-1 to cleave pro-IL-1 $\beta$. The released IL-1 $\beta$ aggravates the inflammatory response and eventually contributes to the pathogenesis of OA. 
Since their discovery, CtBPs have been known as transcriptional corepressors [32]. Previous studies have indicated that CtBPs consistently function as corepressors in different cancers [32]. In terms of molecular mechanisms, overexpressed CtBPs can form transcriptional complexes with numerous transcription factors to inhibit the expression of multiple tumor suppressor genes [32]. However, it seems that CtBPs can function as either corepressors or coactivators in other diseases and biological processes [36-38]. Biochemically, they also form a complex with HATs (or HDACs) and transcription factors to regulate gene transcription [28, 29, 32]. In this study, we comprehensively verified the activation of NLRP3 by the CPAC transcriptional machinery using a variety of experimental methods. To our knowledge, this is the first report to show that CtBPs can regulate the inflammatory response. Given the important role of inflammation in the pathogenesis of many diseases, we speculate that it would also have a similar activation role in the pathogenesis of other inflammation-related diseases. Although $C t B P s$ are overexpressed in many cancers and other diseases, the underlying mechanism of their overexpression is still obscure. Some studies have revealed that miRNAs may be involved in the regulation of $C t B P s$. For example, $\mathrm{Du}$ and colleagues found that miR-485-3p negatively regulates the expression of $C t B P 1$ by directly binding to its 3 -untranslated region (3'-UTR) [46]. Deng and colleagues identified that miR-137 can repress the expression of CtBP1 by binding to its 3'-UTR [39]. In this study, we identified that the overexpression of $C t B P s$ is caused by the decreased DNA methylation levels in their promoters. This new mechanism also provides clues for revealing mechanisms of $C t B P$ overexpression in other biological processes. Several small molecules, such as MTOB, NSC95397 and HIPP (hydroxyimino-3phenylpropanoic acid), have been reported to exhibit strong and specific abilities to block CtBPs [32], which indicates that we can evaluate the role of these small molecules in the treatment of OA.

In addition to our study, many other studies have also identified that DNA methylation is involved in the pathogenesis of $\mathrm{OA}$ by affecting the expression of multiple genes, such as COL2A1 (Collagen type II Alpha 1 chain), COL9A1 (Collagen type IX Alpha 1 chain), COL10A1 (Collagen type X Alpha 1 chain), ACAN (Aggrecan), MMP3, MMP9, MMP13, ADAMTS4 (ADAM Metallopeptidase with thrombospondin type 1 motif) and $\operatorname{IL1B}[47,48]$. In mammalian genomes, DNA methylation is mainly controlled by three DNMTs including DNMT1, DNMT3A and DNMT3B [49]. Although many groups have reported the involvement of DNA methylation in the pathogenesis of OA $[47,48]$, most of them have not examined DNMT mRNA or protein levels in OA biopsies. Sesselmann and colleagues detected the expression of DNMT1 and DNMT3A in OA chondrocytes but did not observe significant changes [50]. Nakano and colleagues measured the protein levels of DNMT1 and DNMT3A in fibroblast-like synoviocytes (FLS) from rheumatoid arthritis (RA) and OA patients [51]. Although they also did not find significant differences in these two proteins between RA and OA samples, they identified that IL-1 $\beta$ stimulation decreased DNMT1 and DNMT3A mRNA levels in FLS [51]. The reason for the difference between our study ( 2-fold decrease in both DNMT1 and DNMT3A mRNA levels) and other groups' results may be because of the sample population and controls. Sesselmann and colleagues only used 10 paired biopsies in their study [50]. Nakano and colleagues compared DNMT1 and DNMT3A protein levels in RA-FLS and OA-FLS and did not use noninflammatory samples as controls [51]. In contrast, we used 48 noninflammatory biopsies and 48 OA samples, which may be more reliable.

To identify CtBP-dependent genes, we conducted a microarray analysis and found multiple overlapping genes that were dependent on both $C t B P 1$ and $C t B P 2$. Except for NLRP3, we also found other overlapping genes such as IL-1B, IL-6, IL15, S100A8 (S100 calcium-binding protein A8), S100A9, PTGS1 (Prostaglandin-endoperoxide synthase 1), TNFA, VCAM1 (Vascular cell adhesion molecule 1), TGFB1, CDH1, Bax, Bim and MMP13 (Supplementary Table-6 and -7). Interestingly, most of these genes including IL-1B, IL-6, IL-15, PTGS1, and VCAM1 are $\mathrm{NF}-\kappa \mathrm{B}$ targets. The activation of NF- $\kappa \mathrm{B}$ signaling is associated with inflammatory diseases, and it has also been reported that NF- $\mathrm{KB}$ signaling is involved in the pathogenesis of OA [23]. Given that NF- $\kappa B$ is a transcription factor family, we speculate that CtBPs may also activate NF-кB-dependent signaling. We are currently investigating whether CtBPs can form a transcriptional complex with NF-kB. In our experiments, a very interesting phenomenon is that the protein levels of OA markers CD31, CD55 and CD68 are dependent on CtBP levels (Supplementary Figure 1). We speculate that one possibility is that these OA markers are downstream targets of $C t B P s$, and the other possibility may be because of the activation proinflammatory cytokines, which further activate OA markers. We will verify these two possibilities in the subsequent studies. The other interesting phenomenon is the mRNA level of $I L-1 B$ was dependent on CtBPs (Figure 2). The activation of NLRP3 only promotes the maturation of IL-1 $\beta$ instead of changing its mRNA level. Thus, we speculate that 
CtBPs may have different roles in the regulation of $I L-1 B$ mRNA and protein levels. As discussed above, there is a possibility that CtBPs form a complex with $N F-\kappa B$ to induce $I L-1 B$ mRNA level.

In summary, our results demonstrate that the DNA methylation mediates the expression of CtBPs. Overexpressed CtBPs form a transcriptional complex with p300 and AP1, whereby the CPAC transcriptional machinery specifically activates NLRP3 expression and its downstream signaling. Our study found for the first time that CtBPs can regulate the inflammatory response. Our results provide new understanding and a breakthrough in the study of inflammation-related diseases.

\section{Supplementary Material}

Supplementary figures and tables. http://www.ijbs.com/v16p0994s1.pdf

\section{Acknowledgments}

We thank all patients who participated in this study and their families for their understanding and support.

\section{Authors' contributions}

J.M. designed the research and experiments. X.S. performed the majority of the experiments including immunoblots, Co-IP, IP, ChIP and qRT-PCR analyses. L.X., J.C., X.C., X. C., S.Y., H.L., and G.Z. performed some of the experiments including clinical sample collection, cell culture, gene overexpression and knockdown, IHC, and IF. J.M. and X.S. analyzed the data, performed statistical analyses and wrote the manuscript. All authors reviewed and approved the manuscript.

\section{Ethical approval statement}

The clinical samples were acquired with written informed consent from all of the participants following protocols approved by the ethical board of Xi'an Jiaotong University College of Medicine. All experimental procedures used in this study were performed in accordance with the approved guidelines of the ethical board of Xi'an Jiaotong University College of Medicine.

\section{Competing Interests}

The authors have declared that no competing interest exists.

\section{References}

1. Chen D, Shen J, Zhao W, Wang T, Han L, Hamilton JL, et al. Osteoarthritis: toward a comprehensive understanding of pathological mechanism. Bone Res. 2017; 5:16044

2. Sokolove J, Lepus CM. Role of inflammation in the pathogenesis of osteoarthritis: latest findings and interpretations. Ther Adv Musculoskelet Dis. 2013; 5:77-94.
3. Neogi T. The epidemiology and impact of pain in osteoarthritis. Osteoarthritis Cartilage. 2013; 21:1145-53.

4. Loeser RF, Goldring SR, Scanzello CR, Goldring MB. Osteoarthritis: a disease of the joint as an organ. Arthritis Rheum. 2012; 64:1697-707.

5. Daheshia M, Yao JQ. The interleukin 1beta pathway in the pathogenesis of osteoarthritis. J Rheumatol. 2008; 35:2306-12.

6. Pearson MJ, Herndler-Brandstetter D, Tariq MA, Nicholson TA, Philp AM, Smith HL, et al. IL-6 secretion in osteoarthritis patients is mediated by chondrocyte-synovial fibroblast cross-talk and is enhanced by obesity. Sci Rep. 2017; 7:3451.

7. Maksymowych WP, Russell AS, Chiu P, Yan A, Jones N, Clare T, et al. Targeting tumour necrosis factor alleviates signs and symptoms of inflammatory osteoarthritis of the knee. Arthritis Res Ther. 2012;14: R206.

8. Raghu $\mathrm{H}$, Lepus $\mathrm{CM}$, Wang $\mathrm{Q}$, Wong $\mathrm{HH}$, Lingampalli $\mathrm{N}$, Oliviero $\mathrm{F}$, et al. CCL2/CCR2, but not CCL5/CCR5, mediates monocyte recruitment, inflammation and cartilage destruction in osteoarthritis. Ann Rheum Dis. 2017;76: 914-22.

9. Alaaeddine N, Antoniou J, Moussa M, Hilal G, Kreichaty G, Ghanem I, et al. The chemokine CCL20 induces proinflammatory and matrix degradative responses in cartilage. Inflamm Res. 2015; 64:721-31.

10. Zhao LR, Xing RL, Wang PM, Zhang NS, Yin SJ, Li XC, et al. NLRP1 and NLRP3 inflammasomes mediate LPS/ATPinduced pyroptosis in knee osteoarthritis. Mol Med Rep. 2018;17: 5463-9.

11. Tetlow LC, Adlam DJ, Woolley DE. Matrix metalloproteinase and proinflammatory cytokine production by chondrocytes of human osteoarthritic cartilage: associations with degenerative changes. Arthritis Rheum. 2001; 44:585-94.

12. Franchi L, Eigenbrod T, Munoz-Planillo R, Nunez G. The inflammasome: a caspase-1-activation platform that regulates immune responses and disease pathogenesis. Nat Immunol. 2009; 10:241-7.

13. Gomez R, Villalvilla A, Largo R, Gualillo O, Herrero-Beaumont G. TLR4 signalling in osteoarthritis--finding targets for candidate DMOADs. Nat Rev Rheumatol. 2015; 11:159-70.

14. Lepetsos P, Papavassiliou AG. ROS/oxidative stress signaling in osteoarthritis. Biochim Biophys Acta. 2016; 1862:576-91.

15. De Santis M, Di Matteo B, Chisari E, Cincinelli G, Angele P, Lattermann C, et al. The Role of Wnt Pathway in the Pathogenesis of OA and Its Potential Therapeutic Implications in the Field of Regenerative Medicine. Biomed Res Int. 2018; 2018:7402947.

16. Lu YC, Yeh WC, Ohashi PS. LPS/TLR4 signal transduction pathway. Cytokine. 2008; 42:145-51.

17. Komiya Y, Habas R. Wnt signal transduction pathways. Organogenesis. 2008; 4:68-75.

18. Ahmed AU, Williams BR, Hannigan GE. Transcriptional Activation of Inflammatory Genes: Mechanistic Insight into Selectivity and Diversity. Biomolecules. 2015; 5:3087-111.

19. Payankaulam S, Li LM, Arnosti DN. Transcriptional repression: conserved and evolved features. Curr Biol. 2010; 20:R764-71.

20. Perissi V, Aggarwal A, Glass CK, Rose DW, Rosenfeld MG. A corepressor/coactivator exchange complex required for transcriptional activation by nuclear receptors and other regulated transcription factors. Cell. 2004; 116:511-26.

21. Bannister AJ, Kouzarides T. Regulation of chromatin by histone modifications. Cell Res. 2011; 21:381-95.

22. Fuda NJ, Ardehali MB, Lis JT. Defining mechanisms that regulate RNA polymerase II transcription in vivo. Nature. 2009; 461:186-92.

23. Rigoglou S, Papavassiliou AG. The NF-kappaB signalling pathway in osteoarthritis. Int J Biochem Cell Biol. 2013;45:2580-4.

24. Fisch KM, Gamini R, Alvarez-Garcia O, Akagi R, Saito M, Muramatsu Y, et al. Identification of transcription factors responsible for dysregulated networks in human osteoarthritis cartilage by global gene expression analysis. Osteoarthritis Cartilage 2018:26:1531-8.

25. Lian K, Zmuda JM, Nevitt MC, Lui L, Hochberg MC, Greene D, et al. Type I collagen alpha1 Sp1 transcription factor binding site polymorphism is associated with reduced risk of hip osteoarthritis defined by severe joint space narrowing in elderly women. Arthritis Rheum. 2005;52:1431-6.

26. Wu YH, Liu W, Zhang L, Liu XY, Wang Y, Xue B, et al. Effects of microRNA-24 targeting C-myc on apoptosis, proliferation, and cytokine expressions in chondrocytes of rats with osteoarthritis via MAPK signaling pathway. J Cell Biochem. 2018;119:7944-58.

27. Barnes PJ, Adcock IM, Ito K. Histone acetylation and deacetylation: importance in inflammatory lung diseases. Eur Respir J. 2005;25:552-63.

28. Sterner DE, Berger SL. Acetylation of histones and transcription-related factors. Microbiol Mol Biol Rev. 2000; 64:435-59.

29. Struhl K. Histone acetylation and transcriptional regulatory mechanisms. Genes Dev. 1998;12: 599-606.

30. Subramanian T, Chinnadurai G. Association of class I histone deacetylases with transcriptional corepressor CtBP. FEBS Lett. 2003;540:255-8.

31. Kim JH, Cho EJ, Kim ST, Youn HD. CtBP represses p300-mediated transcriptional activation by direct association with its bromodomain. Nat Struct Mol Biol. 2005;12:423-8.

32. Blevins MA, Huang M, Zhao R. The Role of CtBP1 in Oncogenic Processes and Its Potential as a Therapeutic Target. Mol Cancer Ther. 2017; 16:981-90. 
33. Dai F, Xuan Y, Jin JJ, Yu S, Long ZW, Cai H, et al. CtBP2 overexpression promotes tumor cell proliferation and invasion in gastric cancer and is associated with poor prognosis. Oncotarget. 2017; 8:28736-49.

34. Xuan Q, Zhong X, Li W, Mo Z, Huang Y, Hu Y. CtBP2 is associated with angiogenesis and regulates the apoptosis of prostate cancer cells. Oncol Rep. 2017; 38:1259-67.

35. Zhang W, Duan N, Zhang O, Song T, Li Z, Chen X, et al. The intracellular $\mathrm{NADH}$ level regulates atrophic nonunion pathogenesis through the CtBP2-p300-Runx2 transcriptional complex. Int J Biol Sci. 2018; 14:2023-36.

36. Paliwal S, Ho N, Parker D, Grossman SR. CtBP2 Promotes Human Cancer Cell Migration by Transcriptional Activation of Tiam1. Genes Cancer. 2012; 3:481-90.

37. Jin W, Scotto KW, Hait WN, Yang JM. Involvement of CtBP1 in the transcriptional activation of the MDR1 gene in human multidrug resistant cancer cells. Biochem Pharmacol. 2007; 74:851-9.

38. Ray SK, Li HJ, Metzger E, Schule R, Leiter AB. CtBP and associated LSD1 are required for transcriptional activation by NeuroD1 in gastrointestinal endocrine cells. Mol Cell Biol. 2014; 34:2308-17.

39. Deng Y, Deng H, Bi F, Liu J, Bemis LT, Norris D, et al. MicroRNA-137 targets carboxyl-terminal binding protein 1 in melanoma cell lines. Int J Biol Sci. 2011; 7:133-7.

40. Kottawatta KS, So KH, Kodithuwakku SP, Ng EH, Yeung WS, Lee KF. MicroRNA-212 Regulates the Expression of Olfactomedin 1 and C-Terminal Binding Protein 1 in Human Endometrial Epithelial Cells to Enhance Spheroid Attachment In vitro. Biol Reprod. 2015; 93:109.

41. McNabb DS, Reed R, Marciniak RA. Dual luciferase assay system for rapid assessment of gene expression in Saccharomyces cerevisiae. Eukaryot Cell. 2005;4:1539-49.

42. Murthy PK, Dennis VA, Lasater BL, Philipp MT. Interleukin-10 modulates proinflammatory cytokines in the human monocytic cell line THP-1 stimulated with Borrelia burgdorferi lipoproteins. Infect Immun. 2000;68:6663-9.

43. Li Q, Li H, Zhao X, Wang B, Zhang L, Zhang C, et al. DNA Methylation Mediated Downregulation of miR-449c Controls Osteosarcoma Cell Cycle Progression by Directly Targeting Oncogene c-Myc. Int J Biol Sci. 2017; 13:1038-50.

44. Bournazou E, Samuels J, Zhou H, Krasnokutsky S, Patel J, Han T, et al. Vascular Adhesion Protein-1 (VAP-1) as Predictor of Radiographic Severity in Symptomatic Knee Osteoarthritis in the New York University Cohort. Int J Mol Sci. 2019;20:E2642.

45. Franchi L, Eigenbrod T, Munoz-Planillo R, Nunez G. The inflammasome: a caspase-1-activation platform that regulates immune responses and disease pathogenesis. Nat Immunol. 2009;10:241-7.

46. Du K, Zhang X, Lou Z, Guo P, Zhang F, Wang B, et al. MicroRNA485-3p negatively regulates the transcriptional co-repressor CtBP1 to control the oncogenic process in osteosarcoma cells. Int J Biol Sci. 2018; 14:1445-56.

47. Cui D, Xu X. DNA Methyltransferases, DNA Methylation, and Age-Associated Cognitive Function. Int J Mol Sci. 2018;19:E1315.

48. den Hollander W, Meulenbelt I. DNA Methylation in Osteoarthritis. Curr Genomics. 2015; 16:419-26.

49. Miranda-Duarte A. DNA Methylation in Osteoarthritis: Current Status and Therapeutic Implications. Open Rheumatol J. 2018; 12:37-49.

50. Sesselmann S, Soder S, Voigt R, Haag J, Grogan SP, Aigner T. DNA methylation is not responsible for p21WAF1/CIP1 down-regulation in osteoarthritic chondrocytes. Osteoarthritis Cartilage. 2009; 17:507-12.

51. Nakano K, Boyle DL, Firestein GS. Regulation of DNA methylation in rheumatoid arthritis synoviocytes. J Immunol. 2013; 190:1297-303. 